\title{
MONODROMY OF FIBER-TYPE ARRANGEMENTS AND ORBIT CONFIGURATION SPACES
}

\author{
DANIEL C. COHEN
}

\begin{abstract}
We prove similar theorems concerning the structure of bundles involving complements of fiber-type hyperplane arrangements and orbit configuration spaces. These results facilitate analysis of the fundamental groups of these spaces, which may be viewed as generalizations of the Artin pure braid group. In particular, we resolve two disparate conjectures. We show that the Whitehead group of the fundamental group of the complement of a fiber-type arrangement is trivial, as conjectured by Aravinda, Farrell, and Rouchon AFR. For the orbit configuration space corresponding to the natural action of a finite cyclic group on the punctured plane, we determine the structure of the Lie algebra associated to the lower central series of the fundamental group. Our results show that this Lie algebra is isomorphic to the module of primitives in the homology of the loop space of a related orbit configuration space, as conjectured by Xicoténcatl $\mathrm{X}$ i].
\end{abstract}

\section{INTRODUCTION}

Let $M$ be a manifold without boundary of dimension at least two. The configuration space of $n$ ordered points in $M$ is the subspace of the product space $M^{n}$ defined by

$$
F(M, n)=\left\{\left(x_{1}, \ldots, x_{n}\right) \in M^{n} \mid x_{i} \neq x_{j} \text { if } i \neq j\right\} .
$$

These spaces arise in numerous contexts, including of course that of braid groups. The symmetric group $\Sigma_{n}$ acts freely on $F(M, n)$. The fundamental group of $F(M, n) / \Sigma_{n}$ is called the (full) braid group of $M$, and that of $F(M, n)$ is the pure braid group of $M$. In the case $M=\mathbb{C}$, these groups are the classical Artin braid groups, see the books of Birman [Bi] and Hansen [Ha], to which we refer as general references on braids.

Let $Q_{n}$ denote a set of $n$ distinct points in $M$. A fundamental property of configuration spaces is given by the following classical result, which we will use extensively.

Theorem (Fadell and Neuwirth [ [FN], Theorem 3). For $\ell \leq n$, the projection onto the first $\ell$ coordinates, $p: F(M, n) \rightarrow F(M, \ell)$, is a locally trivial bundle, with fiber $F\left(M \backslash Q_{\ell}, n-\ell\right)$.

The focus of this paper is on two related generalizations of configuration spaces. First, we study the class of fiber-type arrangements. An arrangement of hyperplanes is a finite collection of codimension one affine subspaces of Euclidean space $\mathbb{C}^{\ell}$. See Orlik and Terao OT as a general reference on arrangements. The complement of an arrangement $\mathcal{A}$ is the manifold $M(\mathcal{A})=\mathbb{C}^{\ell} \backslash \bigcup_{H \in \mathcal{A}} H$. The configuration space $F(\mathbb{C}, n)$ may be realized as the complement in $\mathbb{C}^{n}$ of the braid arrangement consisting of the hyperplanes $H_{i, j}=\operatorname{ker}\left(x_{j}-x_{i}\right), 1 \leq i<j \leq n$. Briefly, an arrangement is fiber-type if, as is the case for the braid arrangement, the complement sits atop a tower of fiber

1991 Mathematics Subject Classification. Primary 20F36, 52B30; Secondary 19B99, 20F40.

Key words and phrases. fiber-type arrangement, orbit configuration space, monomial braid group, strongly poly-free group, Whitehead group, lower central series.

Partially supported by grants LEQSF(1996-99)-RD-A-04 and LEQSF(1999-2002)-RD-A-01 from the Louisiana Board of Regents. 
bundles, the projection maps of which are the restrictions to hyperplane complements of linear maps $\mathbb{C}^{k} \rightarrow \mathbb{C}^{k-1}$.

The second generalization arises in the following way. Let $\Gamma$ be a (finite) group, acting freely on the manifold $M$. The orbit configuration space $F_{\Gamma}(M, n)$ is the subspace of the product space $M^{n}$ consisting of all ordered $n$-tuples $\left(x_{1}, \ldots, x_{n}\right)$ of points in $M$ for which the orbits $\Gamma \cdot x_{i}$ and $\Gamma \cdot x_{j}$ do not intersect for $i \neq j$. These spaces were recently studied by Xicoténcatl [Xi], who showed that, like their classical counterparts, they support certain Lie algebra structures in their loop space homology, and proved a fibration theorem generalizing the Fadell-Neuwirth theorem stated above.

In this paper, we use the Fadell-Neuwirth theorem to determine the structure of certain bundles involving both complements of fiber-type arrangements and orbit configuration spaces. These results, the proofs of which are straightforward and virtually identical, facilitate analysis of the fundamental groups of these spaces. As these spaces generalize configuration spaces, their fundamental groups may be viewed as generalizations of the Artin pure braid group. We pursue several structural aspects of these generalized pure braid groups. Much is already known about a number of these groups.

For a fiber-type arrangement $\mathcal{A}$, the group $G=\pi_{1}(M(\mathcal{A}))$ is an "almost direct product" of free groups. That is, the group $G$ may be realized as an iterated semidirect product of free groups, the factors of which act on one another by conjugation. In [FR1], Falk and Randell use this structure to prove that fiber-type arrangement groups satisfy the celebrated LCS formula, relating the Betti numbers and the ranks of the lower central series quotients of these groups. For the Artin pure braid group, this result was obtained by other means by Kohno K2]. Using the almost direct product structure of the group $G$ of a fiber-type arrangement, one can also construct a finite free resolution of the integers over the group ring $\mathbb{Z} G$ and show that $G$ is of type FL CS2], show that $G$ is residually nilpotent [FR2], orderable [Pa], etc..

Our results further reveal the structure of these groups. From the relationship with configuration spaces we establish, it follows that the conjugation action in the almost direct product structure is given by pure braid automorphisms. Using this, we show that fiber-type arrangement groups are strongly poly-free, see Definition 1.2.1. Aravinda, Farrell, and Roushon AFR have recently shown that the Whitehead group of any strongly poly-free group is trivial. Thus, the Whitehead group of a fiber-type arrangement group is trivial, as conjectured in AFR.

In principle, we give an algorithm for presenting the group of a fiber-type arrangement as an almost direct product. Using a natural generalization of the techniques for computing the braid monodromy of a complex line arrangement developed in [CS1], we provide a method for calculating the pure braids which dictate the structure of fibertype arrangement groups. We illustrate the method using the Coxeter arrangement of type $\mathrm{B}$, giving a presentation of the Brieskorn generalized pure braid group $P B_{n}$ which exhibits the iterated semidirect product structure of this group.

The complex reflection arrangements associated to full monomial groups provide a bridge between the two generalizations of configuration spaces we consider. The complement of such an arrangement may be realized as the orbit configuration space $F_{\Gamma}\left(\mathbb{C}^{*}, n\right)$, where $\Gamma$ is a finite cyclic group, acting on $\mathbb{C}^{*}$ by multiplication by a primitive root of unity. By combining the above methods with known results on fundamental groups of hyperplane complements, we also obtain a presentation for the fundamental group of this orbit configuration space. This presentation is used to study the Lie algebra associated to the lower central series of this "pure monomial braid group," revealing that the structure of this Lie algebra is as conjectured by Xicoténcatl [Xi].

Conventions. Denote by $\operatorname{Aut}(G)$ the group of right automorphisms of a group $G$, with multiplication $\alpha \cdot \beta=\beta \circ \alpha$. For $u, v \in G$, write $u^{v}=v^{-1} u v$ and $[u, v]=u v u^{-1} v^{-1}$. 


\section{Fiber-Type ArRangements}

\subsection{Monodromy of Fiber-Type Arrangements.}

In this section, we identify the monodromy of a strictly linearly fibered arrangement. When iteratively applied to fiber-type arrangements, this identification gives the iterated semidirect product structure of the fundamental group of the complement of such an arrangement. We first recall the definitions of these arrangements, see [FR1, OT].

Definition 1.1.1. A hyperplane arrangement $\mathcal{A}$ in $\mathbb{C}^{\ell+1}$ is strictly linearly fibered if there is a choice of coordinates $(\mathbf{x}, z)=\left(x_{1}, \ldots, x_{\ell}, z\right)$ on $\mathbb{C}^{\ell+1}$ so that the restriction, $p$, of the projection $\mathbb{C}^{\ell+1} \rightarrow \mathbb{C}^{\ell},(\mathbf{x}, z) \mapsto \mathbf{x}$, to the complement $M(\mathcal{A})$ is a fiber bundle projection, with base $p(M(\mathcal{A}))=M(\mathcal{B})$, the complement of an arrangement $\mathcal{B}$ in $\mathbb{C}^{\ell}$, and fiber the complement of finitely many points in $\mathbb{C}$. We say $\mathcal{A}$ is strictly linearly fibered over $\mathcal{B}$.

Definition 1.1.2. An arrangement $\mathcal{A}=\mathcal{A}_{1}$ of finitely many points in $\mathbb{C}^{1}$ is fiber-type. An arrangement $\mathcal{A}=\mathcal{A}_{\ell}$ of hyperplanes in $\mathbb{C}^{\ell}$ is fiber-type if $\mathcal{A}$ is strictly linearly fibered over a fiber-type arrangement $\mathcal{A}_{\ell-1}$ in $\mathbb{C}^{\ell-1}$.

The complement of a fiber-type arrangement sits atop a tower of fiber bundles

$$
M\left(\mathcal{A}_{\ell}\right) \stackrel{p_{\ell}}{\longrightarrow} M\left(\mathcal{A}_{\ell-1}\right) \stackrel{p_{\ell-1}}{\longrightarrow} \cdots \stackrel{p_{2}}{\longrightarrow} M\left(\mathcal{A}_{1}\right)=\mathbb{C} \backslash\left\{d_{1} \text { points }\right\},
$$

where the fiber of $p_{k}$ is homeomorphic to the complement of $d_{k}$ points in $\mathbb{C}$. Repeated application of the homotopy exact sequence of a bundle shows that $M\left(\mathcal{A}_{\ell}\right)$ is a $K(\pi, 1)$ space, where $\pi=\pi_{1}\left(M\left(\mathcal{A}_{\ell}\right)\right)$. The integers $\left\{d_{1}, \ldots, d_{\ell}\right\}$ are called the exponents of the fiber-type arrangement $\mathcal{A}$. In general, an arrangement $\mathcal{A}$ is said to be $K(\pi, 1)$ if the complement $M(\mathcal{A})$ is an Eilenberg-MacLane space of type $K(\pi, 1)$.

Remark 1.1.3. The rank of an arrangement $\mathcal{A}$ is the largest number of linearly independent hyperplanes in $\mathcal{A}$. A useful alternative definition of a fiber-type, or supersolvable, arrangement is given in [FT: An (affine) arrangement $\mathcal{A}$ is supersolvable if there is a sequence $\mathcal{A}=\mathcal{A}_{\ell} \supseteq \cdots \supseteq \mathcal{A}_{1}$ such that $\operatorname{rank} \mathcal{A}_{j+1}=\operatorname{rank} \mathcal{A}_{j}+1$ for each $j$, and for distinct $H, H^{\prime} \in \mathcal{A}_{j}$ with $H \cap H^{\prime} \neq \emptyset$, there exists $H^{\prime \prime} \in \mathcal{A}_{i}$ with $i<j$ and $H \cap H^{\prime} \subset H^{\prime \prime}$.

Example 1.1.4. Consider the braid arrangement, $\left\{\operatorname{ker}\left(y_{i}-y_{j}\right), 1 \leq i<j \leq n\right\}$, in $\mathbb{C}^{n}$, with complement $F(\mathbb{C}, n)=\left\{\mathbf{y} \in \mathbb{C}^{n} \mid y_{i} \neq y_{j}\right.$ if $\left.i \neq j\right\}$, the configuration space of $n$ ordered points in $\mathbb{C}$, where $\mathbf{y}=\left(y_{1}, \ldots, y_{n}\right)$. The braid arrangement is the prototypical example of a fiber-type arrangement. By the Fadell-Neuwirth theorem, projection onto the first $n$ coordinates yields a bundle $p_{n+1}: F(\mathbb{C}, n+1) \rightarrow F(\mathbb{C}, n)$, with fiber the complement of $n$ points in $\mathbb{C}$.

As noted previously, the fundamental groups of $F(\mathbb{C}, n)$ and $F(\mathbb{C}, n) / \Sigma_{n}$ are the classical Artin braid groups $P_{n}$ and $B_{n}$, see [Bi, Ha. We record presentations of these groups. The full braid group has presentation

$$
B_{n}=\left\langle\sigma_{i}(1 \leq i<n) \mid \begin{array}{c}
\sigma_{i} \sigma_{i+1} \sigma_{i}=\sigma_{i+1} \sigma_{i} \sigma_{i+1}(1 \leq i<n-1) \\
\sigma_{i} \sigma_{j}=\sigma_{j} \sigma_{i}(|j-i|>1)
\end{array}\right\rangle .
$$

The bundle of configuration spaces $p_{n+1}: F(\mathbb{C}, n+1) \rightarrow F(\mathbb{C}, n)$ admits a section $s: F(\mathbb{C}, n) \rightarrow F(\mathbb{C}, n+1)$, given by $s(\mathbf{y})=(\mathbf{y}, z)$, where $z=\left(1+\sum_{i=1}^{n}\left|y_{i}\right|^{2}\right)^{1 / 2}$. From this and the homotopy sequence of the bundle, it follows that the pure braid group admits the structure of an iterated semidirect product of free groups, $P_{n}=$ $\mathbb{F}_{n-1} \rtimes_{\alpha_{n-1}} \cdots \rtimes_{\alpha_{2}} \mathbb{F}_{1}$. The monodromy homomorphism $\alpha_{k}: P_{k} \rightarrow \operatorname{Aut}\left(\mathbb{F}_{k}\right)$ is the 
restriction to $P_{k}$ of the Artin representation $\alpha_{k}: B_{k} \rightarrow \operatorname{Aut}\left(\mathbb{F}_{k}\right)$, defined by

$$
\alpha_{k}\left(\sigma_{i}\right)\left(t_{j}\right)= \begin{cases}t_{i} t_{i+1} t_{i}^{-1} & \text { if } j=i, \\ t_{i} & \text { if } j=i+1, \\ t_{j} & \text { otherwise, }\end{cases}
$$

where $\mathbb{F}_{k}=\left\langle t_{1}, \ldots, t_{k}\right\rangle$. The standard presentation of the pure braid group exhibits the iterated semidirect product structure. The group $P_{n}$ has generators

$$
A_{i, j}=\sigma_{j-1} \cdots \sigma_{i+1} \sigma_{i}^{2} \sigma_{i+1}^{-1} \cdots \sigma_{j-1}^{-1} \quad(1 \leq i<j \leq n),
$$

and, for $j<l$, defining relations

$$
A_{i, j}^{-1} A_{k, l} A_{i, j}= \begin{cases}\left(A_{i, l} A_{j, l}\right) A_{k, l}\left(A_{i, l} A_{j, l}\right)^{-1} & \text { if } k=i \text { or } k=j, \\ {\left[A_{i, l} A_{j, l}\right] A_{k, l}\left[A_{i, l} A_{j, l}\right]^{-1}} & \text { if } i<k<j, \\ A_{k, l} & \text { otherwise. }\end{cases}
$$

We now identify the monodromy of a strictly linearly fibered arrangement. Suppose $\mathcal{A}$ is strictly linearly fibered over $\mathcal{B}$, and write $|\mathcal{B}|=m$ and $|\mathcal{A}|=m+n$. It then follows from the definition that a defining polynomial for $\mathcal{A}$ factors as

$$
Q(\mathcal{A})=Q(\mathcal{B}) \cdot \phi(\mathbf{x}, z)
$$

where $Q(\mathcal{B})=Q(\mathcal{B})(\mathbf{x})$ is a defining polynomial for $\mathcal{B}$, and $\phi(\mathbf{x}, z)$ is a product of $n$ linear functions:

$$
\phi(\mathbf{x}, z)=\left(z-g_{1}(\mathbf{x})\right)\left(z-g_{2}(\mathbf{x})\right) \cdots\left(z-g_{n}(\mathbf{x})\right), \quad g_{j}(\mathbf{x}) \text { linear. }
$$

Since $\phi(\mathbf{x}, z)$ has distinct roots for any $\mathbf{x} \in M(\mathcal{B})$, the associated root map

$$
g: M(\mathcal{B}) \rightarrow \mathbb{C}^{n}, \quad g(\mathbf{x})=\left(g_{1}(\mathbf{x}), g_{2}(\mathbf{x}), \ldots, g_{n}(\mathbf{x})\right),
$$

takes values in the configuration space $F(\mathbb{C}, n)$.

Theorem 1.1.5. Let $\mathcal{B}$ be an arrangement of $m$ hyperplanes, and let $\mathcal{A}$ be an arrangement of $m+n$ hyperplanes which is strictly linearly fibered over $\mathcal{B}$. Then the bundle $p: M(\mathcal{A}) \rightarrow M(\mathcal{B})$ is equivalent to the pullback of the bundle of configuration spaces $p_{n+1}: F(\mathbb{C}, n+1) \rightarrow F(\mathbb{C}, n)$ along the map $g$.

Proof. Denote points in $F(\mathbb{C}, n+1)$ by $(\mathbf{y}, z)$, where $\mathbf{y}=\left(y_{1}, \ldots, y_{n}\right) \in F(\mathbb{C}, n)$ and $z \in \mathbb{C}$ satisfies $z \neq y_{j}$ for each $j$. Similarly, denote points in $M(\mathcal{A})$ by $(\mathbf{x}, z)$, where $\mathbf{x} \in M(\mathcal{B})$ and $\phi(\mathbf{x}, z) \neq 0$. Then $p_{n+1}(\mathbf{y}, z)=\mathbf{y}$ and $p(\mathbf{x}, z)=\mathbf{x}$.

Let $E=\{(\mathbf{x},(\mathbf{y}, z)) \in M(\mathcal{B}) \times F(\mathbb{C}, n+1) \mid g(\mathbf{x})=\mathbf{y}\}$ be the total space of the pullback of $p_{m+1}: F(\mathbb{C}, n+1) \rightarrow F(\mathbb{C}, n)$ along $g$. It is then readily checked that the map $M(\mathcal{A}) \rightarrow E$ defined by $(\mathbf{x}, z) \mapsto(\mathbf{x},(g(\mathbf{x}), z))$ is an equivalence of bundles.

Denote the fundamantal group of the complement of an arrangement $\mathcal{A}$ by $G(\mathcal{A})$, or simply by $G$ if the underlying arrangement is clear. We record some immediate consequences of the above result.

Corollary 1.1.6. Let $\mathcal{B}$ be an arrangement of $m$ hyperplanes, and let $\mathcal{A}$ be an arrangement of $m+n$ hyperplanes which is strictly linearly fibered over $\mathcal{B}$. Then,

1. the bundle $p: M(\mathcal{A}) \rightarrow M(\mathcal{B})$ admits a section;

2. the structure group of the bundle $p: M(\mathcal{A}) \rightarrow M(\mathcal{B})$ is the pure braid group $P_{n}$;

3. the monodromy of the bundle $p: M(\mathcal{A}) \rightarrow M(\mathcal{B})$ factors as $\eta=\alpha_{n} \circ \gamma$, where $\alpha_{n}: P_{n} \rightarrow \operatorname{Aut}\left(\mathbb{F}_{n}\right)$ is the Artin representation and $\gamma=g_{*}: G(\mathcal{B}) \rightarrow P_{n}$ is the map on fundamental groups induced by $g$; and

4. if $\mathcal{B}$ is $K(\pi, 1)$, then $\mathcal{A}$ is $K(\pi, 1)$ and the group $G(\mathcal{A})$ is isomorphic to the semidirect product $\mathbb{F}_{n} \rtimes_{\eta} G(\mathcal{B})$. 
Remark 1.1.7. Let $g_{i}$ be the homotopy class of a meridional loop about the hyperplane $H_{i}$ of $\mathcal{B}$. The classes $g_{i}$ generate the group $G(\mathcal{B})$, see OT]. Suppose $\mathcal{A}$ is strictly linearly fibered over $\mathcal{B}$, and identify the fundamental group of the fiber of $p: M(\mathcal{A}) \rightarrow M(\mathcal{B})$ with the free group $\mathbb{F}_{n}=\left\langle t_{1}, \ldots, t_{n}\right\rangle$. If $\mathcal{B}$ is $K(\pi, 1)$, then so is $\mathcal{A}$, and $1 \rightarrow \mathbb{F}_{n} \rightarrow$ $G(\mathcal{A}) \leftrightarrows G(\mathcal{B}) \rightarrow 1$ is split exact. Identify $\mathbb{F}_{n}$ and $G(\mathcal{B})$ with their images in $G(\mathcal{A})$ under the inclusion and splitting respectively. Then the group $G(\mathcal{A})$ has presentation

$$
G(\mathcal{A})=\left\langle g_{i}(1 \leq i \leq m), t_{j}(1 \leq j \leq n) \mid g_{i}^{-1} t_{j} g_{i}=\eta\left(g_{i}\right)\left(t_{j}\right)(1 \leq j \leq n, 1 \leq i \leq m)\right\rangle .
$$

The automorphism $\eta\left(g_{i}\right)$ is obtained by applying the Artin representation (1.2) to the pure braid $\gamma\left(g_{i}\right)$. A method for calculating the braids $\gamma\left(g_{i}\right)$ is presented in Section 1.3.

Now let $\mathcal{A}$ be a fiber-type arrangement in $\mathbb{C}^{\ell}$ with exponents $\left\{d_{1}, \ldots, d_{\ell}\right\}$. Then there is a choice of coordinates $\left(x_{1}, \ldots, x_{\ell}\right)$ on $\mathbb{C}^{\ell}$ so that a defining polynomial for $\mathcal{A}$ factors as $Q(\mathcal{A})=\prod_{k=1}^{\ell} Q_{k}\left(x_{1}, \ldots, x_{k}\right)$, where $\operatorname{deg} Q_{k}=d_{k}$ for each $k$, see (1.5). For each $j \leq \ell$, the polynomial $\prod_{k=1}^{j} Q_{k}$ defines a fiber-type arrangement $\mathcal{A}_{j}$ in $\mathbb{C}^{j}$ with exponents $\left\{d_{1}, \ldots, d_{j}\right\}$, and $\mathcal{A}_{j}$ is strictly linearly fibered over $\mathcal{A}_{j-1}$.

Identify the fundamental group of the fiber of the bundle $p_{j}: M\left(\mathcal{A}_{j}\right) \rightarrow M\left(\mathcal{A}_{j-1}\right)$ with the free group $\mathbb{F}_{d_{j}}$ on $d_{j}$ generators for each $j$. The action of the group $G\left(\mathcal{A}_{j-1}\right)$ on $\mathbb{F}_{d_{j}}$ is the composition, $\eta_{j}:=\alpha_{d_{j}} \circ \gamma_{j}$, of the Artin representation $\alpha_{d_{j}}: P_{d_{j}} \rightarrow \operatorname{Aut}\left(\mathbb{F}_{d_{j}}\right)$ and the homomorphism $\gamma_{j}: G\left(\mathcal{A}_{j-1}\right) \rightarrow P_{d_{j}}$ induced by the map $g_{j}$ of (1.6). Repeated application of Theorem 1.1.5 and Corollary 1.1.6 yields

Theorem 1.1.8. The fundamental group $G\left(\mathcal{A}_{\ell}\right)$ of the complement of the fiber-type arrangement $\mathcal{A}_{\ell}$ admits the structure of an iterated semidirect product of free groups

$$
G\left(\mathcal{A}_{\ell}\right)=\mathbb{F}_{d_{\ell}} \rtimes_{\eta_{\ell}} \cdots \rtimes \mathbb{F}_{d_{2}} \rtimes_{\eta_{2}} \mathbb{F}_{d_{1}}
$$

with (split) pure braid extensions $\eta_{j}: G\left(\mathcal{A}_{j-1}\right) \rightarrow P_{d_{j}}<\operatorname{Aut}\left(\mathbb{F}_{d_{j}}\right)$.

Remark 1.1.9. For $1 \leq j \leq \ell$, fix generators $x_{q, j}, 1 \leq q \leq d_{j}$, for the free group $\mathbb{F}_{d_{j}}$. Then the group $G=G\left(\mathcal{A}_{\ell}\right)$ has presentation

$$
G=\left\langle x_{q, j}\left(1 \leq q \leq d_{j}, 1 \leq j \leq \ell\right) \mid x_{p, i}^{-1} x_{q, j} x_{p, i}=\eta_{j}\left(x_{p, i}\right)\left(x_{q, j}\right)(i<j)\right\rangle .
$$

\subsection{Fiber-Type Arrangement Groups are Strongly Poly-Free.}

As noted in the Introduction, the structure of the fundamental group of the complement of a fiber-type arrangement exhibited in Theorem 1.1.8 may be used to obtain a number of interesting and important consequences. In this section we record another, showing that these groups are strongly poly-free. We first recall the definition of this class of groups from AFR.

Definition 1.2.1. A discrete group $G$ is strongly poly-free if there exists a finite filtration of $G$ by subgroups, $1=G_{0}<G_{1}<\cdots<G_{\ell}=G$, which satisfies the conditions:

(1) $G_{k}$ is normal in $G$ for each $k$;

(2) $G_{k+1} / G_{k}$ is a finitely generated free group; and

(3) for each $w \in G$ and each $k$, there is a compact surface $\Sigma$ with non-empty boundary and a diffeomorphism $f: \Sigma \rightarrow \Sigma$ such that the induced homomorphism $f_{*}$ on $\pi_{1}(\Sigma)$ is equal to $\operatorname{conj}_{w}$ in $\operatorname{Out}\left(\pi_{1}(\Sigma)\right)$, where $\operatorname{conj}_{w}$ is the action of $w$ on $G_{k+1} / G_{k}$ by conjugation and $\pi_{1}(\Sigma)$ is identified with $G_{k+1} / G_{k}$ via a suitable isomorphism.

Theorem 1.2.2 (Aravinda, Farrell, and Roushon AFR, Theorem 2.1). For each n, the pure braid group $P_{n}$ is strongly poly-free.

In light of this result, and those of Section 1.1, it is natural to speculate that the fundamental group of the complement of any fiber-type arrangement is strongly polyfree. This is indeed the case. 
Theorem 1.2.3. Let $\mathcal{A}=\mathcal{A}_{\ell}$ be a fiber-type arrangement. Then the fundamental group $G=G\left(\mathcal{A}_{\ell}\right)$ of the complement is strongly poly-free.

Proof. From Theorem 1.1.8, we have $G \cong \mathbb{F}_{d_{\ell}} \rtimes_{\eta_{\ell}} \cdots \rtimes_{\eta_{3}} \mathbb{F}_{d_{2}} \rtimes_{\eta_{2}} \mathbb{F}_{d_{1}}$. For $1 \leq k \leq \ell$, let $G_{k}=\mathbb{F}_{d_{\ell}} \rtimes_{\eta_{\ell}} \cdots \rtimes_{\eta_{\ell-k+2}} \mathbb{F}_{d_{\ell-k+1}}$. Then $G_{k}$ is normal in $G$ and $G_{k+1} / G_{k}=\mathbb{F}_{d_{\ell-k}}$ is free, so conditions (1) and (2) of the definition are satisfied.

We show that condition (3) holds by induction on the cohomological dimension of $G$, which we may assume without loss of generality is equal to $\ell$. In the case $\ell=1, G=\mathbb{F}_{d}$ is a (single) finitely generated free group acting on itself by conjugation, and condition (3) clearly holds. In general, from Theorem 1.1.5 we have a commuting diagram

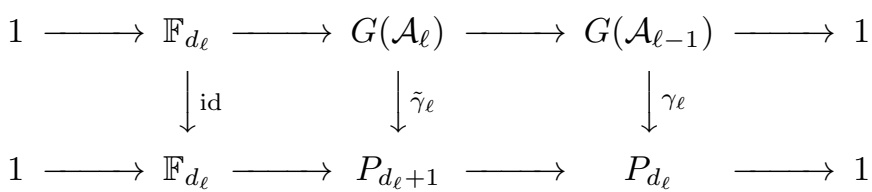

where $\gamma_{\ell}$ is induced by the map $g_{\ell}: M\left(\mathcal{A}_{\ell-1}\right) \rightarrow F\left(\mathbb{C}, d_{\ell}\right)$ from $(1.6)$, and $\tilde{\gamma}_{\ell}$ is induced by $\tilde{g}_{\ell}: M\left(\mathcal{A}_{\ell}\right) \rightarrow F\left(\mathbb{C}, d_{\ell}+1\right)$ defined by $\tilde{g}_{\ell}(\mathbf{x}, z)=\left(g_{\ell}(\mathbf{x}), z\right)$. From Corollary 1.1.6, we have $G\left(\mathcal{A}_{\ell}\right)=\mathbb{F}_{d_{\ell}} \rtimes_{\eta_{\ell}} G\left(\mathcal{A}_{\ell-1}\right)$, where $\eta_{\ell}=\alpha_{d_{\ell}} \circ \gamma_{\ell}$ and $\alpha_{d_{\ell}}: P_{d_{\ell}} \rightarrow \operatorname{Aut}\left(\mathbb{F}_{d_{\ell}}\right)$ is the Artin representation. Using the semidirect product structure, every element $w \in G\left(A_{\ell}\right)$ may be expressed as $w=u v$, where $u \in \mathbb{F}_{d_{\ell}}$ and $v \in G\left(\mathcal{A}_{\ell-1}\right)$.

For $w \in G\left(\mathcal{A}_{\ell}\right)$, consider the conjugation action of $w$ on $G_{k+1} / G_{k}$. In the case $k=0$, for $a \in G_{1}=\mathbb{F}_{d_{\ell}}$ and $w=u v$ as above, we have

$$
w^{-1} a w=v^{-1} u^{-1} a u v=\eta_{\ell}(v)\left(u^{-1} a u\right)=\gamma_{\ell}(v)^{-1} u^{-1} a u \gamma_{\ell}(v) .
$$

Thus in this instance, conjugation by $w$ coincides with conjugation by the pure braid $u \cdot \gamma_{\ell}(v)=\tilde{\gamma}_{\ell}(u v)=\tilde{\gamma}_{\ell}(w) \in P_{d_{\ell}+1}$. So for $k=0$, condition (3) holds by the result of Aravinda, Farrell, and Roushon stated in Theorem 1.2.2 above.

For the case $k>0$, let $a \in G\left(\mathcal{A}_{\ell-1}\right)$ and consider $w^{-1} a w$. In this instance, we have

$$
w^{-1} a w=v^{-1} u^{-1} a u v=v^{-1}\left(u^{-1} a u a^{-1}\right) v \cdot v^{-1} a v .
$$

Now $v^{-1}\left(u^{-1} a u a^{-1}\right) v$ is in $G_{1}$, since $u \in G_{1}$ and $G_{1}$ is normal in $G$. Consequently, the conjugation action of $w=u v$ on $G_{k+1} / G_{k}$ coincides with that of $v \in G\left(\mathcal{A}_{\ell-1}\right)$ for $k>0$. So condition (3) holds by induction in this case.

In [AFR, Theorem 1.3], it is shown that the Whitehead group of the direct product of a strongly poly-free group $G$ and a free abelian group is trivial, $W h\left(G \times \mathbb{Z}^{m}\right)=0$ for every $m \geq 0$. This result and Theorem 1.2 .3 above yield the following, which resolves positively the conjecture of Aravinda, Farrell, and Roushon stated in AFR, Section 2].

Corollary 1.2.4. Let $G$ be the fundamental group of the complement of a fiber-type arrangement $\mathcal{A}$. Then the Whitehead group of $G \times \mathbb{Z}^{m}$ is trivial for every $m \geq 0$.

Remark 1.2.5. For $G=G(\mathcal{A})$ as above, note that the group $G \times \mathbb{Z}^{m}$ may itself be realized as the fundamental group of the complement of the fiber-type arrangement $\mathcal{A} \times \mathcal{B}$, where $\mathcal{B}$ is the arrangement of coordinate hyperplanes in $\mathbb{C}^{m}$.

\subsection{Calculating the Monodromy.}

In this section, we present a method for calculating the monodromy of the bundle $p: M(\mathcal{A}) \rightarrow M(\mathcal{B})$ for an arrangement $\mathcal{A}$ of $m+n$ hyperplanes, strictly linearly fibered over the arrangement $\mathcal{B}$ of $m$ hyperplanes. This technique may be applied repeatedly to determine the iterated semidirect product structure of the group of a fiber-type arrangement, as we illustrate in the next section. Since the monodromy factors as $\eta=$ $\alpha_{n} \circ \gamma: G(\mathcal{B}) \rightarrow P_{n} \rightarrow \operatorname{Aut}\left(\mathbb{F}_{n}\right)$, where $\alpha_{n}: P_{n} \rightarrow \operatorname{Aut}\left(\mathbb{F}_{n}\right)$ is the Artin representation, we focus on the determination of the homomorphism $\gamma: G(\mathcal{B}) \rightarrow P_{n}$ induced by the 
map $g: M(\mathcal{B}) \rightarrow F(\mathbb{C}, m)$ from (1.6). As this is a natural generalization of the method for finding the braid monodromy of a complex line arrangement developed in [CS1, we call $\gamma: G(\mathcal{B}) \rightarrow P_{n}$ the braid monodromy of the bundle $p: M(\mathcal{A}) \rightarrow M(\mathcal{B})$.

Write $\mathcal{B}=\left\{H_{1}, \ldots, H_{m}\right\}$, and let $L$ be a complex line in $\mathbb{C}^{\ell}$ that is transverse to $\mathcal{B}$. Denote the coordinate on $L$ by $x$, and the point $L \cap H_{j}$ by $q_{j}$. Then $C:=L \backslash\left\{q_{1}, \ldots, q_{m}\right\}$ is the complement of $m=|\mathcal{B}|$ points in $L=\mathbb{C}$, and $\pi_{1}(C)=\mathbb{F}_{m}$. Let $i: C \hookrightarrow M(\mathcal{B})$ denote the natural inclusion, and let $\widehat{g}=g \circ i: C \rightarrow F(\mathbb{C}, n)$ denote the restriction to $C$ of the map $g: M(\mathcal{B}) \rightarrow F(\mathbb{C}, n)$. Passing to fundamantal groups, we have $\widehat{g}_{*}=\widehat{\gamma}=\gamma \circ i_{*}: \mathbb{F}_{m} \rightarrow G(\mathcal{B}) \rightarrow P_{n}$. Since $i_{*}$ is surjective, it suffices to determine the homomorphism $\hat{\gamma}: \mathbb{F}_{m} \rightarrow P_{n}$.

The pullback of the configuration space bundle $F(\mathbb{C}, n+1) \rightarrow F(\mathbb{C}, n)$ along $\widehat{g}$ is equivalent to the restriction, $\widehat{p}: Y \rightarrow C$, of the bundle $p: M(\mathcal{A}) \rightarrow M(\mathcal{B})$, where

$$
Y=\{(x, z) \in C \times \mathbb{C} \mid \phi(x, z) \neq 0\}=\left\{(x, z) \in \mathbb{C}^{2} \mid \prod_{j=1}^{n}\left(x-q_{j}\right) \cdot \phi(x, z) \neq 0\right\},
$$

and $\phi(x, z)$ is the restriction of $\phi(\mathbf{x}, z)$ to $L$. The polynomial $\phi(x, z)$ defines an arrangement $\mathcal{H}$ of $n$ lines in $\mathbb{C}^{2}$. The multiple points of $\mathcal{H}$ necessarily lie on the lines $x=q_{j}$. Note that more than one such multiple point may lie on a given such line, and that there may be lines $x=q_{j}$ upon which no such multiple points lie. The present construction generalizes that of CS1] in these senses.

Order the $m$ distinct points $q_{j}$ in $L=\mathbb{C}$ by decreasing real part, breaking ties by imaginary part. If $i<j$, then $\operatorname{Re}\left(q_{i}\right)>\operatorname{Re}\left(q_{j}\right)$, or $\operatorname{Re}\left(q_{i}\right)=\operatorname{Re}\left(q_{j}\right)$ and $\operatorname{Im}\left(q_{i}\right)<\operatorname{Im}\left(q_{j}\right)$. Fix a basepoint $q_{0}$ in $C$ with $\operatorname{Re}\left(q_{0}\right)>\operatorname{Re}\left(q_{1}\right)$, and let $\xi=\xi(t)$ be a path in $\mathbb{C}$, emanating from $q_{0}$ and passing through the ordered points $q_{j}$. In a small disk $D_{\epsilon}\left(q_{j}\right)$ about $q_{j}$, take $\xi$ to be a horizontal line segment, which passes through $q_{j}$ from right to left as $t$ increases, and let $q_{j}^{\prime}=q_{j}-\epsilon$ and $q_{j}^{\prime \prime}=q_{j}+\epsilon$. Choose $\epsilon>0$ small, so that $q_{i} \notin D_{\epsilon}\left(q_{j}\right)$ for $i \neq j$. Let $\xi_{j, j+1}$ be the portion of $\xi$ from $q_{j}^{\prime \prime}$ to $q_{j+1}^{\prime}$, let $\xi_{j}^{\prime}$ be the portion of the boundary of the disk $D_{\epsilon}\left(q_{j}\right)$ from $q_{j}^{\prime}$ to $q_{j}^{\prime \prime}$, and $\xi_{j}^{\prime \prime}$ the portion of $\partial D_{\epsilon}\left(q_{j}\right)$ from $q_{j}^{\prime \prime}$ to $q_{j}^{\prime}$ (both oriented counterclockwise).

Let $u_{j}$ denote the homotopy class of the loop in $C$ based at $q_{0}$ which traverses the paths $\xi_{i, i+1}$ and $\xi_{i}^{\prime}$ for $i<j$ in the natural order, passes around $q_{j}$ along $\xi_{j}^{\prime}$ and $\xi_{j}^{\prime \prime}$, and returns to $q_{0}$ along the $\xi_{i, i+1}$ and $\xi_{i}^{\prime}$ with $i<j$. Using these meridians, identify $\pi_{1}\left(C, q_{0}\right)$ with $\mathbb{F}_{m}=\left\langle u_{1}, \ldots, u_{m}\right\rangle$. The monodromy of the bundle $\widehat{p}: Y \rightarrow C$ is determined by the (pure) braids $\widehat{\gamma}\left(u_{j}\right)$. Since the images $v_{j}=i_{*}\left(u_{j}\right)$ generate $G(\mathcal{B})=\pi_{1}(M(\mathcal{B})$ ), these braids also determine the monodromy of $p: M(\mathcal{A}) \rightarrow M(\mathcal{B})$.

The braids $\widehat{\gamma}\left(u_{j}\right)=\gamma\left(v_{j}\right)$ may be calculated from the braided wiring diagram $\mathcal{W}=$ $\{(x, z) \in \xi \times \mathbb{C} \mid \phi(x, z)=0\}$ associated to the path $\xi$, cf. [CS1, Section 5]. For $q \neq q_{j}$, $1 \leq j \leq m$, the set $\mathcal{W} \cap\{x=q\}$ consists of $n$ distinct points, the intersections of the lines of $\mathcal{H}$ with $\{x=q\}$. Order the lines of $\mathcal{H}$ by increasing real part of the $n$ points of $\mathcal{W} \cap\left\{x=q_{0}\right\}$, breaking ties as above. Let $[n]=\{1, \ldots, n\}$. The diagram $\mathcal{W}$ may be (abstractly) specified by a sequence of partitions of $[n]$ and braids,

$$
\mathcal{W}=\mathcal{W}_{m}=\left\{I(1), \beta_{1,2}, I(2), \beta_{2,3}, \ldots, \beta_{m-1, m}, I(m), \beta_{m, m+1}\right\} .
$$

The braids $\beta_{i, i+1}$ are elements of the full braid group $B_{n}$, obtained by tracing the components of $\mathcal{W}$ over the path $\xi_{i, i+1}$, see CS1, Section 4.4]. The partitions

$$
I(j)=\left(I_{1}(j)\left|I_{2}(j)\right| \cdots \mid I_{r}(j)\right)=\left(1, \ldots, j_{1}\left|j_{1}+1, \ldots, j_{2}\right| \cdots, \mid j_{r-1}+1, \ldots, n\right)
$$

record the ordering at $x=q_{j}^{\prime}$ of the lines of $\mathcal{H}$ which meet at $x=q_{j}$. If no lines of $\mathcal{H}$ meet at $x=q_{j}$, then $I(j)=(1|2| \cdots \mid n)$ consists of $n$ singletons.

To each block $I_{k}=\{i, i+1, \ldots, i+s\}$ of such a partition $I$, we associate a permutation braid $\mu_{I_{k}}$, a half twist on $I_{k}$, given in terms of the standard generators of $B_{n}$ by

$$
\mu_{I_{k}}=\left(\sigma_{i} \cdots \sigma_{i+s-1}\right)\left(\sigma_{i} \cdots \sigma_{i+s-2}\right) \cdots\left(\sigma_{i} \cdots \sigma_{i+1}\right)\left(\sigma_{i}\right) .
$$


If $\left|I_{k}\right|=1$, set $\mu_{I_{k}}=1$. Note that $\mu_{I_{k}}$ and $\mu_{I_{k^{\prime}}}$ commute for $k \neq k^{\prime}$, and that the product $\Upsilon_{I}=\mu_{I_{1}} \cdots \mu_{I_{r}}$ records the braiding of the components of $\mathcal{W}$ over each of the paths $\xi_{j}^{\prime}$ and $\xi_{j}^{\prime \prime}$. The local (braid) monodromy around the point $q_{j}$ is given by $\Upsilon_{I}^{2}$, the product of the full twists $\mu_{I_{k}}^{2}$. The braid monodromy $\widehat{\gamma}: \mathbb{F}_{m} \rightarrow P_{n}$ is then given by

$$
\widehat{\gamma}\left(u_{j}\right)=\beta_{j}^{-1} \Upsilon_{I(j)}^{2} \beta_{j},
$$

where the conjugating braids $\beta_{j}$ satisfy $\beta_{1}=1$ and $\beta_{j+1}=\beta_{j, j+1} \cdot \Upsilon_{I(j)} \cdot \beta_{j}$ for $j \geq 1$.

We express the braid monodromy solely in terms of pure braids, cf. [CS1, Section 5.3]. Recall the original ordering of the lines of $\mathcal{H}$ at the basepoint $x=q_{0}$. Let $V(j)=$ $\left(V_{1}(j)\left|V_{2}(j)\right| \cdots \mid V_{r}(j)\right)$ be the partition of $[n]$ recording the indices of these lines meeting at $x=q_{j}$ in terms of this ordering. To a block $V_{k}=\left\{k_{1}, \ldots, k_{s}\right\}$ of such a partition (with $k_{i}<k_{i+1}$ ), associate the full twist on $V_{k}$, given in terms of the standard generators of $P_{n}$ by

$$
A_{V_{k}}=\left(A_{k_{1}, k_{2}}\right) \cdot\left(A_{k_{1}, k_{3}} A_{k_{2}, k_{3}}\right) \cdots \cdots\left(A_{k_{1}, k_{s}} \cdots A_{k_{s-1}, k_{s}}\right) .
$$

Geometrically, these braids are obtained by gathering the strands indexed by $V_{k}$ together behind the remaining strands, performing a full twist on the $V_{k}$ strands, and then returning these strands to their original positions. If $s=1$, set $A_{V_{k}}=1$. Expressing (1.7) in terms of pure braids yields

Theorem 1.3.1. The braid monodromy $\widehat{\gamma}: \mathbb{F}_{m} \rightarrow P_{n}$ of the bundle $\widehat{p}: Y \rightarrow C$, and hence that of the strictly linearly fibered bundle $p: M(\mathcal{A}) \rightarrow M(\mathcal{B})$, is given by

$$
\gamma \circ i_{*}\left(u_{j}\right)=\widehat{\gamma}\left(u_{j}\right)=\prod_{k=1}^{r} A_{V_{k}(j)}^{\zeta_{k}}=\prod_{k=1}^{r} \zeta_{k}^{-1} \cdot A_{V_{k}(j)} \cdot \zeta_{k},
$$

where $\zeta_{k} \in P_{n}$ is determined by the subdiagram $\mathcal{W}_{j-1}$ of $\mathcal{W}$ and the block $V_{k}(j)$.

Remark 1.3.2. If $\mathcal{A}$ is strictly linearly fibered over $\mathcal{B}$, the associated braid monodromy determines the rank two elements of the intersection poset $L(\mathcal{A})$ in the following way. Order the hyperplanes of $\mathcal{A} \backslash \mathcal{B}=\mathcal{H}=\left\{\mathcal{H}_{1}, \ldots, \mathcal{H}_{n}\right\}$ as indicated above. Let $g \in G(\mathcal{B})$ be a meridian about a hyperplane $H$ of $\mathcal{B}$, with associated monodromy generator $\gamma(g)=\prod_{k=1}^{r} A_{V_{k}}^{\zeta_{k}}$. Then the blocks $V_{k}$ record those hyperplanes of $\mathcal{A} \backslash \mathcal{B}$ which meet $H$ in codimension two. In other words, for each $k$, we have $H \cap\left(\bigcap_{j \in V_{k}} \mathcal{H}_{j}\right) \in L_{2}(\mathcal{A})$. Thus, $L_{2}(\mathcal{A})$ consists of elements of this form, together with elements of $L_{2}(\mathcal{B})$.

In addition to recording combinatorial information, the expression (1.8) of the braid monodromy also sheds light on the presentations of strictly linearly fibered and fibertype arrangement groups noted in Remarks 1.1.7 and 1.1.9. To this end, we briefly describe the behavior of pure braids of the form (1.8) under the Artin representation. Note that the factors of these braids commute, $\left[A_{V_{k}(j)}^{\zeta_{k}}, A_{V_{l}(j)}^{\zeta_{l}}\right]=1$. For $t \in \mathbb{F}_{n}$, let $\tilde{t}$ denote some conjugate of $t$. Let $V=\left(V_{1}\left|V_{2}\right| \cdots \mid V_{r}\right)$ be a partition of [n]. If $V_{k}=\left\{k_{1}, \ldots, k_{s}\right\}$ is a block of $V$, set $\widetilde{t}_{V_{k}}=\widetilde{t}_{k_{1}} \cdots \widetilde{t}_{k_{s}}$.

Proposition 1.3.3. Let $\gamma=\prod_{k=1}^{r} A_{V_{k}}^{\zeta_{k}}$ be a braid with commuting factors associated to the partition $V$ of $[n]$, and let $\alpha_{n}: P_{n} \rightarrow \operatorname{Aut}\left(\mathbb{F}_{n}\right)$ be the Artin representation. Then

$$
\alpha_{n}(\gamma)\left(t_{j}\right)=\widetilde{t}_{V_{k}} \cdot \widetilde{t}_{j} \cdot \widetilde{t}_{V_{k}}^{-1},
$$

where $V_{k}$ is the unique block of $V$ containing $j$.

Proof. Write $\gamma=B \cdot A_{V_{k}}^{\zeta_{k}}$, where $j \in V_{k}$ and $B=\prod_{l \neq k} A_{V_{l}}^{\zeta_{l}}$, and identify a pure braid with its image under the Artin representation. Then $\alpha_{n}(\gamma)\left(t_{j}\right)=\gamma\left(t_{j}\right)=A_{V_{k}}^{\zeta_{k}} \circ B\left(t_{j}\right)$. The action of $P_{n}<\operatorname{Aut}\left(\mathbb{F}_{n}\right)$ is by conjugation, so $\gamma\left(t_{j}\right)=\zeta_{k} \circ A_{V_{k}}\left(w \cdot t_{j} \cdot w^{-1}\right)$, where $w \cdot t_{j} \cdot w^{-1}=\zeta_{k}^{-1} \circ B\left(t_{j}\right)$. A calculation with the Artin representation reveals that $A_{V_{k}}\left(t_{j}\right)=t_{V_{k}} \cdot t_{j} \cdot t_{V_{k}}^{-1}$ for $j \in V_{k}$. So we have $\gamma\left(t_{j}\right)=v \cdot \zeta_{k}\left(t_{V_{k}} \cdot t_{j} \cdot t_{V_{k}}^{-1}\right) \cdot v^{-1}$, where $v=\zeta_{k} \circ A_{V_{k}}(w)$, and the result follows. 


\subsection{The Coxeter Arrangement of type B.}

Let $\mathcal{B}_{n}$ denote the Coxeter arrangement of type $\mathrm{B}$ in $\mathbb{C}^{n}$, with defining polynomial

$$
Q\left(\mathcal{B}_{n}\right)=x_{1} \cdots x_{n} \prod_{i<j}\left(x_{j}^{2}-x_{i}^{2}\right),
$$

and complement $M\left(\mathcal{B}_{n}\right)$. As shown by Brieskorn $[\mathrm{Br}]$, this arrangement is fiber-type, and the fundamental group of $M\left(\mathcal{B}_{n}\right)$ is the pure braid group of type $\mathrm{B}, G\left(\mathcal{B}_{n}\right)=P B_{n}$. We illustrate the method described in the previous section by determining the iterated semidirect product structure of this generalized pure braid group.

Denote the $n^{2}$ hyperplanes of $\mathcal{B}_{n}$ by $H_{i}=\operatorname{ker}\left(x_{i}\right)$ and $H_{i, j}^{ \pm}=\operatorname{ker}\left(x_{j} \pm x_{i}\right)$. The line $L=\left\{\left(x, 2 x+b_{2}, \ldots, n x+b_{n}\right)\right\}$, where $b_{k}=(2 k+1)$ !, is transverse to $\mathcal{B}_{n}$. Write $L \cap H_{i}=h_{i}$ and $L \cap H_{i, j}^{ \pm}=h_{i, j}^{ \pm}$. Notice that these points are real, and check that

$$
h_{n-1, n}^{-}<\cdots<h_{1, n}^{-}<h_{n}<h_{1, n}^{+}<\cdots<h_{n-1, n}^{+}<\cdots \cdots<h_{1,2}^{-}<h_{2}<h_{1,2}^{+}<h_{1} .
$$

With this notation, we have $C=L \backslash\left\{h_{i}\right\} \cup\left\{h_{i, j}^{ \pm}\right\}$.

The arrangement $\mathcal{B}_{n+1}$ is strictly linearly fibered over $\mathcal{B}_{n}$, and has defining polynomial $Q\left(\mathcal{B}_{n+1}\right)=Q\left(\mathcal{B}_{n}\right) \cdot \phi_{n}(\mathbf{x}, z)$, where

$$
\phi_{n}(\mathbf{x}, z)=\left(z+x_{n}\right) \cdots\left(z+x_{1}\right) \cdot z \cdot\left(z-x_{1}\right) \cdots\left(z-x_{n}\right) .
$$

Let $g_{n}: M\left(\mathcal{B}_{n}\right) \rightarrow F(\mathbb{C}, 2 n+1)$ be the associated root map (cf. 1.6)), inducing $\gamma_{n}: P B_{n} \rightarrow P_{2 n+1}$ on fundamental groups, and let $\widehat{g}_{n}$ denote the restriction of $g_{n}$ to $C$. The restriction, $\phi_{n}(x, z)$, of $\phi_{n}(\mathbf{x}, z)$ to $L$ defines an arrangement $\mathcal{H}^{n}$ of $2 n+1$ lines in $\mathbb{C}^{2}$. These lines have real defining equations, so let $q_{0} \in C$ be a real basepoint with $h_{1}<q_{0}$, and let $\xi=\left[d, q_{0}\right]$ be a line segment along the real axis in $L$ with $d<h_{n-1, n}^{-}$. The resulting wiring diagram $\mathcal{B} \mathcal{W}^{n}$ is unbraided: the braids $\beta_{i, i+1}=1$ are all trivial. The arrangement $\mathcal{H}^{2}$ and diagram $\mathcal{B W}^{3}$ are depicted in Figure 1.

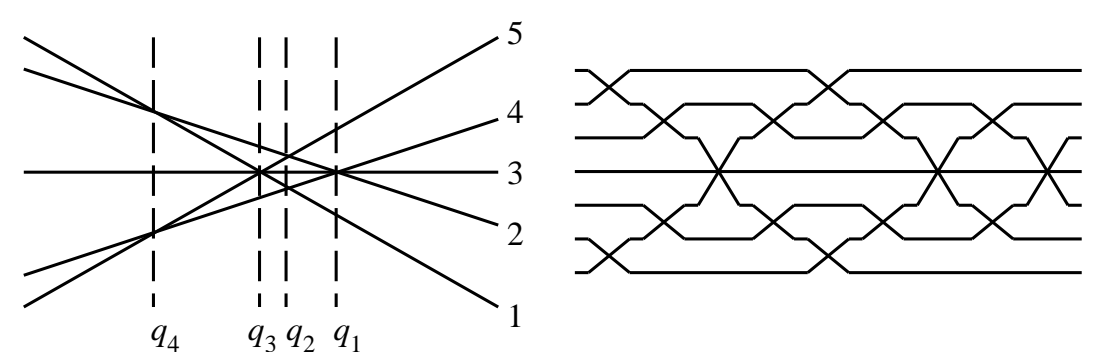

Figure 1. Type B wiring diagrams for $n=2$ (left) and $n=3$ (right)

Example 1.4.1. We explicitly carry out the monodromy calculation in the case $n=2$. Write $q_{1}=h_{1}, q_{2}=h_{1,2}^{-}, q_{3}=h_{2}$, and $q_{4}=h_{1,2}^{+}$. Refering to Figure 1, we see that the partitions $I(j)$ and associated braids $\Upsilon_{I(j)}$ are

$$
\begin{aligned}
& I(1)=I(3)=(1|2,3,4| 5), \quad \Upsilon_{I(1)}=\Upsilon_{I(3)}=1 \cdot \sigma_{2} \sigma_{3} \sigma_{2} \cdot 1, \\
& I(2)=I(4)=(1,2|3| 4,5), \quad \Upsilon_{I(2)}=\Upsilon_{I(4)}=\sigma_{1} \cdot 1 \cdot \sigma_{4} .
\end{aligned}
$$

Thus the braids $\beta_{j}$ appearing in (1.7) are $\beta_{1}=1, \beta_{2}=\sigma_{2} \sigma_{3} \sigma_{2}, \beta_{3}=\sigma_{1} \sigma_{4} \sigma_{2} \sigma_{3} \sigma_{2}$, and $\beta_{4}=\sigma_{2} \sigma_{3} \sigma_{2} \sigma_{1} \sigma_{4} \sigma_{2} \sigma_{3} \sigma_{2}$.

Write $\pi_{1}(C)=\mathbb{F}_{4}=\left\langle u_{1}, u_{2}, u_{3}, u_{4}\right\rangle$ as before. Combing the braid as in (1.8), the braid monodromy $\widehat{\gamma}_{2}: \mathbb{F}_{4} \rightarrow P_{5}$ is given by

$$
\begin{aligned}
& \widehat{\gamma}_{2}\left(u_{1}\right)=A_{2,3,4} \text {, } \\
& \widehat{\gamma}_{2}\left(u_{3}\right)=A_{1,2} A_{1,3,5} A_{1,2}^{-1} \text {, } \\
& \widehat{\gamma}_{2}\left(u_{2}\right)=A_{1,2} A_{1,3} A_{1,4} A_{1,3}^{-1} A_{1,2}^{-1} \cdot A_{2,5}, \\
& \widehat{\gamma}_{2}\left(u_{4}\right)=A_{1,2} \cdot A_{4,5} \text {. }
\end{aligned}
$$


These braids act on $\mathbb{F}_{5}=\left\langle t_{1}, \ldots, t_{5}\right\rangle$ via the Artin representation $\alpha_{5}: P_{5} \rightarrow \operatorname{Aut}\left(\mathbb{F}_{5}\right)$. Write $\widehat{\eta}_{2}=\alpha_{5} \circ \widehat{\gamma}_{2}$. A calculation using (1.2) yields $\widehat{\eta}_{2}\left(u_{i}\right)\left(t_{j}\right)=w_{i, j} t_{j} w_{i, j}^{-1}$, where

$$
\begin{aligned}
& w_{1, j}=\left\{\begin{array}{ll}
1, & \text { if } j=1,5, \\
t_{2} t_{3} t_{4} & \text { if } j=2,3,4,
\end{array} \quad w_{2, j}= \begin{cases}t_{1} t_{2} t_{3} t_{4} t_{3}^{-1} t_{2}^{-1} & \text { if } j=1, \\
t_{2} t_{5} & \text { if } j=2,5, \\
{\left[t_{2}, t_{5}\right]} & \text { if } j=3, \\
{\left[t_{2}, t_{5}\right] t_{3}^{-1} t_{2}^{-1} t_{1} t_{2} t_{3}} & \text { if } j=4,\end{cases} \right. \\
& w_{3, j}=\left\{\begin{array}{ll}
t_{1} t_{2} t_{3} t_{5} t_{2}^{-1} & \text { if } j=1, \\
1, & \text { if } j=2, \\
t_{2}^{-1} t_{1} t_{2} t_{3} t_{5} & \text { if } j=3,5, \\
{\left[t_{2}^{-1} t_{1} t_{2} t_{3}, t_{5}\right]} & \text { if } j=4,
\end{array} \quad w_{4, j}= \begin{cases}t_{1} t_{2} & \text { if } j=1,2, \\
1 & \text { if } j=3, \\
t_{4} t_{5} & \text { if } j=4,5 .\end{cases} \right.
\end{aligned}
$$

The braid monodromy $\widehat{\gamma}_{2}: \mathbb{F}_{4} \rightarrow P_{5}$ descends to $\gamma_{2}: P B_{2} \rightarrow P_{5}$. With the above Artin representation calculation, this realizes the group $P B_{3}$ as a semidirect product, $P B_{3}=\mathbb{F}_{5} \rtimes_{\eta_{2}} P B_{2}$. We momentarily defer further discussion of this realization.

In general, the group $P B_{n}$ is generated by the images of the generators $u_{j}$ of $\pi_{1}(C)$ under the map induced by inclusion $i: C \hookrightarrow M\left(\mathcal{B}_{n}\right)$. These images, $c_{j}, a_{i, j}$, and $b_{i, j}$, are homotopy classes of meridional loops about the hyperplanes $H_{j}, H_{i, j}^{-}$, and $H_{i, j}^{+}$ respectively. So for instance, $c_{1}=i_{*}\left(u_{1}\right), b_{1,2}=i_{*}\left(u_{2}\right), c_{2}=i_{*}\left(u_{3}\right)$, and $a_{1,2}=i_{*}\left(u_{4}\right)$. The structure of the wiring diagram $\mathcal{B W}^{n}$ is analogous to those exhibited in the examples shown in Figure 1. Analysis of this structure and the ensuing braid monodromy calculation are left to the reader. Define $U_{r, s} \in P_{2 n+1}$ by $U_{r, s}=A_{r, r+1} A_{r, r+2} \cdots A_{r, s}$. The result is

Proposition 1.4.2. The braid monodromy $\gamma: P B_{n} \rightarrow P_{2 n+1}$ is given by

$$
\begin{aligned}
\gamma\left(c_{j}\right) & =U_{n-j+1, n} A_{n-j+1, n+1, n+j+1} U_{n-j+1, n}^{-1} \\
\gamma\left(a_{i, j}\right) & =A_{n+i+1, n+j+1} \cdot U_{n-j+1, n-i} A_{n-j+1, n-i+1} U_{n-j+1, n-i}^{-1}, \\
\gamma\left(b_{i, j}\right) & =A_{n-i+1, n+j+1} \cdot U_{n-j+1, n+i} A_{n-j+1, n+i+1} U_{n-j+1, n+i}^{-1} .
\end{aligned}
$$

As in the case $n=2$ above, applying the Artin representation yields the semidirect product structure of $P B_{n+1}=\mathbb{F}_{2 n+1} \rtimes_{\eta_{n}} P B_{n}$. The correspondence between the generators $t_{i}$ of $\mathbb{F}_{2 n+1}$ and the meridional generators of $P B_{n+1}$ is

$$
t_{i}= \begin{cases}b_{n-i+1, n+1} & \text { if } 1 \leq i \leq n \\ c_{n+1} & \text { if } i=n+1 \\ a_{i-n-1, n+1} & \text { if } n+2 \leq i \leq 2 n+1\end{cases}
$$

Carrying out the aforementioned Artin representation calculations for $k=1, \ldots, n-1$ yields a presentation of the group $P B_{n}$ which exhibits the iterated semidirect product structure. We suppress these lengthy calculations, and state the result below. For $i<j$, let $\bar{a}_{i, j}=a_{1, j} a_{2, j} \cdots a_{i-1, j}$ and $\bar{b}_{i, j}=b_{i-1, j} \cdots b_{2, j} b_{1, j}$, and write $\hat{b}_{i, j}=b_{i, j}^{\bar{b}_{i, j}}$.

Theorem 1.4.3. The Brieskorn generalized pure braid group admits the structure of an iterated semidirect product of free groups, $P B_{n}=\mathbb{F}_{2 n-1} \rtimes \cdots \rtimes \mathbb{F}_{3} \rtimes \mathbb{F}_{1}$, where $\mathbb{F}_{2 j-1}=\left\langle c_{j}, a_{i, j}, b_{i, j}(1 \leq i<j)\right\rangle$. For $j<l$, the action of $\mathbb{F}_{2 j-1}$ on $\mathbb{F}_{2 l-1}$ is given by

$$
\begin{aligned}
& a_{i, j}^{-1} a_{k, l} a_{i, j}=p_{k} a_{k, l} p_{k}^{-1}, \quad a_{i, j}^{-1} b_{k, l} a_{i, j}=q_{k} b_{k, l} q_{k}^{-1}, \quad a_{i, j}^{-1} c_{l} a_{i, j}=c_{l}, \\
& b_{i, j}^{-1} a_{k, l} b_{i, j}=u_{k} a_{k, l} u_{k}^{-1}, \quad b_{i, j}^{-1} b_{k, l} b_{i, j}=v_{k} b_{k, l} v_{k}^{-1}, \quad b_{i, j}^{-1} c_{l} b_{i, j}=w_{l} c_{l} w_{l}^{-1}, \\
& c_{j}^{-1} a_{k, l} c_{j}=x_{k} a_{k, l} x_{k}^{-1}, \quad c_{j}^{-1} b_{k, l} c_{s}=y_{k} b_{k, l} y_{k}^{-1}, \quad c_{j}^{-1} c_{l} c_{j}=z c_{l} z^{-1} \text {, }
\end{aligned}
$$


where $w_{l}=\left[b_{i, l}, a_{j, l}\right], y_{j}=b_{j, l} \bar{b}_{j, l} c_{j} a_{j, l} \bar{b}_{j, l}^{-1}, y_{k}=1$ for $k \neq j, z=\hat{b}_{j, l} c_{l} a_{j, l}$ and

$$
\begin{aligned}
& p_{k}=\left\{\begin{array}{ll}
a_{i, l} a_{j, l} & k=i, j, \\
{\left[a_{i, l}, a_{j, l}\right]} & i<k<j, \\
1 & \text { else, }
\end{array} \quad q_{k}=\left\{\begin{array}{ll}
\hat{b}_{j, l}^{\bar{b}_{r+1, j}^{-1}} & k=i, \\
b_{j, l} \hat{b}_{i, l}^{-1} & k=j, \\
1 & \text { else, }
\end{array} \quad x_{k}= \begin{cases}{\left[\hat{b}_{j, l} c_{l}, a_{j, l}\right]} & k<j, \\
\hat{b}_{j, l} c_{l} & k=j, \\
1 & \text { else, }\end{cases} \right.\right. \\
& u_{k}=\left\{\begin{array}{ll}
{\left[b_{i, l}, a_{j, l}\right]} & k<i \text { or } i<k<j, \\
{\left[b_{i, l}, a_{j, l}\right] \hat{b}_{j, l}^{c_{l} \bar{a}_{i, l}}} & \text { if } k=i, \\
b_{i, l} & k=j, \\
1 & \text { else, }
\end{array} \quad v_{k}= \begin{cases}{\left[b_{i, l}, a_{j, l}\right]} & k<i, \\
b_{i, l} a_{j, l} & k=i, \\
b_{j, l}\left[\bar{b}_{j, l} c_{l} \bar{a}_{i, l}, a_{i, l}\right] a_{i, l} & k=j, \\
1 & \text { else. }\end{cases} \right.
\end{aligned}
$$

Remark 1.4.4. In Le, Section 3.8], Leibman obtains a presentation of the group $P B_{n}$ by different means. We have verified that this presentation and that of Theorem 1.4.3 are equivalent for small $n$. We have not been able to carry out this verification in general, as there appears to be a typographical error in $[\mathrm{LE}]$ affecting the general case.

\section{Orbit Configuration Spaces}

\subsection{Orbit Configuration Space Bundles.}

Let $M$ be a manifold without boundary, and let $\Gamma$ be a finite group which acts freely on $M$. The orbit configuration space consists of all ordered $n$-tuples of points in $M$ which lie in distinct orbits:

$$
F_{\Gamma}(M, n)=\left\{\left(x_{1}, \ldots, x_{n}\right) \in M^{n} \mid \Gamma \cdot x_{i} \cap \Gamma \cdot x_{j}=\emptyset \text { if } i \neq j\right\} .
$$

Let $Q_{n}^{\Gamma}$ denote the union of $n$ distinct orbits, $\Gamma \cdot x_{1}, \ldots, \Gamma \cdot x_{n}$, in $M$. In [Xi], Xicoténcatl proves the following theorem, a natural generalization to orbit configuration spaces of the Fadell-Neuwirth theorem stated in the Introduction.

Theorem 2.1.1 (Xicoténcatl [Xi], Theorem 2.2.2). For $\ell \leq n$, the projection onto the first $\ell$ coordinates, $p_{\Gamma}: F_{\Gamma}(M, n) \rightarrow F_{\Gamma}(M, \ell)$, is a locally trivial bundle, with fiber $F_{\Gamma}\left(M \backslash Q_{\ell}^{\Gamma}, n-\ell\right)$.

The proof given in $[\mathrm{Xi}$ is a natural adaptation of that of $[\overrightarrow{\mathrm{FN}}]$ for classical configuration spaces. For the special case $n-\ell=1$, we give here a different proof, similar to that of Theorem 1.1.5, which sheds light on the structure of these bundles.

Suppose that the order of the finite group $\Gamma$ is $r$, and define a map from the orbit configuration space to the classical configuration space by sending a $n$-tuple of points in $M$ to their orbits. Explicitly, define $f: F_{\Gamma}(M, k) \rightarrow F(M, r n)$ by $f\left(x_{1}, \ldots, x_{n}\right)=$ $\left(\Gamma \cdot x_{1}, \ldots, \Gamma \cdot x_{n}\right)$.

Theorem 2.1.2. The orbit configuration space bundle $p_{\Gamma}: F_{\Gamma}(M, n+1) \rightarrow F_{\Gamma}(M, n)$ is equivalent to the pullback of the bundle $p_{r n+1}: F(M, r n+1) \rightarrow F(M, r n)$ of classical configuration spaces along the map $f$.

Proof. Denote points in $F(M, r n+1)$ by $(\mathbf{y}, z)$, where $\mathbf{y}=\left(y_{1}, \ldots, y_{r n}\right) \in F(M, r n)$ and $z \in M$ satisfies $z \neq y_{j}$ for each $j$. Similarly, denote points in $F_{\Gamma}(M, n+1)$ by $(\mathbf{x}, z)$, where $\mathbf{m}=\left(x_{1}, \ldots, x_{n}\right) \in F_{\Gamma}(M, n)$ and $z \in M$ satisfies $\Gamma \cdot z \cap \Gamma \cdot x_{j}=\emptyset$ for each $j$. Then $p_{r n+1}(\mathbf{y}, z)=\mathbf{y}$ and $p_{\Gamma}(\mathbf{x}, z)=\mathbf{x}$.

Let $E=\left\{(\mathbf{x},(\mathbf{y}, z)) \in F_{\Gamma}(M, n) \times F(M, r n+1) \mid f(\mathbf{x})=\mathbf{y}\right\}$ be the total space of the pullback of $p_{r n+1}: F(M, r n+1) \rightarrow F(M, r n)$ along $f$. It is then readily checked that the map $F_{\Gamma}(M, n+1) \rightarrow E$ defined by $(\mathbf{x}, w) \mapsto(\mathbf{x},(f(\mathbf{x}), w))$ is an equivalence of bundles. 
We are mainly interested in the case where the finite cyclic group $\Gamma=\mathbb{Z} / r \mathbb{Z}$ acts freely on the manifold $M=\mathbb{C}^{*}=\mathbb{C} \backslash\{0\}$ by multiplication by the primitive $r$-th root of unity $\zeta=\exp (2 \pi \sqrt{-1} / r)$. In this instance, Theorem 1.1.5 provides a useful alternative to the above result. This orbit configuration space is given by

$$
F_{\Gamma}\left(\mathbb{C}^{*}, n\right)=\left\{\left(x_{1}, \ldots, x_{n}\right) \in\left(\mathbb{C}^{*}\right)^{n} \mid x_{j} \neq \zeta^{p} x_{i} \text { for } i \neq j \text { and } 1 \leq p \leq r\right\},
$$

and thus may be realized as as the complement in $\mathbb{C}^{n}$ of the arrangement $\mathcal{A}_{r, n}$ consisting of the hyperplanes $H_{j}=\operatorname{ker}\left(x_{j}\right), 1 \leq j \leq n$, and $H_{i, j}^{(p)}=\operatorname{ker}\left(x_{j}-\zeta^{p} x_{i}\right), 1 \leq i<j \leq n$, $1 \leq p \leq r$. These are the reflecting hyperplanes of the full monomial group $G(r, n)$, the complex reflection group isomorphic to the wreath product of the symmetric group $\Sigma_{n}$ and $\Gamma=\mathbb{Z} / r \mathbb{Z}$, so we call $\mathcal{A}_{r, n}$ the (full) monomial arrangement. The projection $p_{\Gamma}: F_{\Gamma}\left(\mathbb{C}^{*}, n\right) \rightarrow F_{\Gamma}\left(\mathbb{C}^{*}, n-1\right)$ reveals the fiber-type structure of this arrangement: $\mathcal{A}_{r, k+1}$ is strictly linearly fibered over $\mathcal{A}_{r, k}$ for $1 \leq k<n$. For the arrangement $\mathcal{A}_{r, k+1}$, the root map $g_{k}: F_{\Gamma}\left(\mathbb{C}^{*}, k\right) \rightarrow F(\mathbb{C}, r k+1)$ of $(1.6)$ is given by

$$
g_{k}\left(x_{1}, \ldots, x_{k}\right)=\left(0, \zeta x_{1}, \ldots, \zeta^{r} x_{1}, \zeta x_{2}, \ldots, \zeta^{r} x_{2}, \ldots, \zeta x_{k}, \ldots, \zeta^{r} x_{k}\right) .
$$

We explicitly record the results of Theorems 1.1.5 and 1.1.8 in this special case.

Theorem 2.1.3. For $\Gamma=\mathbb{Z} / r \mathbb{Z}$ acting freely on $\mathbb{C}^{*}$, the orbit configuration space bundle $p_{\Gamma}: F_{\Gamma}\left(\mathbb{C}^{*}, n+1\right) \rightarrow F_{\Gamma}\left(\mathbb{C}^{*}, n\right)$ is equivalent to the pullback of the classical configuration space bundle $p_{r n+2}: F(\mathbb{C}, r n+2) \rightarrow F(\mathbb{C}, r n+1)$ along the map $g_{n}$. Consequently,

1. the bundle $p_{\Gamma}: F_{\Gamma}\left(\mathbb{C}^{*}, n+1\right) \rightarrow F_{\Gamma}\left(\mathbb{C}^{*}, n\right)$ admits a section;

2. the structure group of the bundle $p_{\Gamma}: F_{\Gamma}\left(\mathbb{C}^{*}, n+1\right) \rightarrow F_{\Gamma}\left(\mathbb{C}^{*}, n\right)$ is the Artin pure braid group on $r n+1$ strands $P_{r n+1}$;

3. the monodromy of $p_{\Gamma}: F_{\Gamma}\left(\mathbb{C}^{*}, n+1\right) \rightarrow F_{\Gamma}\left(\mathbb{C}^{*}, n\right)$ factors as $\alpha_{r n+1} \circ \gamma_{n}$, where $\gamma_{n}: \pi_{1}\left(F_{\Gamma}\left(\mathbb{C}^{*}, n\right)\right) \rightarrow P_{r n+1}$ is the map on fundamental groups induced by $g_{n}$, and $\alpha_{r n+1}: P_{r n+1} \rightarrow \operatorname{Aut}\left(\mathbb{F}_{r n+1}\right)$ is the Artin representation;

4. the arrangement $\mathcal{A}_{r, n}$ is $K(\pi, 1)$, and the group $\pi_{1}\left(F_{\Gamma}\left(\mathbb{C}^{*}, n\right)\right)=\rtimes_{j=1}^{n} \mathbb{F}_{r(j-1)+1}$ admits the structure of an iterated semidirect product of free groups.

\subsection{Pure Monomial Braid Groups.}

In this section, we investigate the structure of the fundamental group of the orbit configuration space $F_{\Gamma}\left(\mathbb{C}^{*}, n\right)$, where $\Gamma=\mathbb{Z} / r \mathbb{Z}$. Since this space is the complement of the reflection arrangement associated to the monomial group $G(r, n)$, we call this fundamental group the pure monomial braid group, and write $P(r, n)=\pi_{1}\left(F_{\Gamma}\left(\mathbb{C}^{*}, n\right)\right)$.

We first construct some geometric braids in the orbit configuration space $F_{\Gamma}\left(\mathbb{C}^{*}, n\right)$. A classical braid on $n$ strands may be described as (an equivalence class of) the motion of $n$ distinct points in the plane through time. Thus a braid $\beta$ may be represented by a collection of $n$ maps $\beta(t)=\left(b_{1}(t), \ldots, b_{n}(t)\right), b_{j}:[0,1] \rightarrow \mathbb{C}$, which satisfy $\beta(0)=\beta(1)$ (as sets), and $b_{i}(t) \neq b_{j}(t)$ for all $t$ if $i \neq j$. If $b_{j}(0)=b_{j}(1)$ for each $j$, the braid $\beta$ is pure, and represents an element of the fundamental group of the configuration space $F(\mathbb{C}, n)$. We adapt these ideas to the orbit configuration space $F_{\Gamma}\left(\mathbb{C}^{*}, n\right)$.

The root map $g_{n}: F_{\Gamma}\left(\mathbb{C}^{*}, n\right) \rightarrow F(\mathbb{C}, r n+1)$ of (2.1) is an imbedding, and the orbit configuration space $F_{\Gamma}\left(\mathbb{C}^{*}, n\right)$ is homeomorphic to the section $S(r, n)$ of $F(\mathbb{C}, r n+1)$ defined by the image of $g_{n}$. If $\beta$ is a braid in the configuration space $F(\mathbb{C}, r n+1)$, call $\beta$ a monomial braid if $\beta(t) \in S(r, n)$ for all $t$. We give two relevant examples.

Define monomial braids $\rho_{i}=\rho_{i}(t)$ by

$$
\begin{aligned}
\rho_{0} & =g_{n} \circ(\exp (2 \pi t \sqrt{-1} / r), 2,3, \ldots, n), \text { and } \\
\rho_{i} & =g_{n} \circ(1, \ldots, i-1, i-\exp (\pi t \sqrt{-1}), i+1+\exp (\pi t \sqrt{-1}), i+2, \ldots, n)
\end{aligned}
$$

for $1 \leq i<n$. Pictures of these braids, for small $r$, are given in Figures 2 and 3 . 

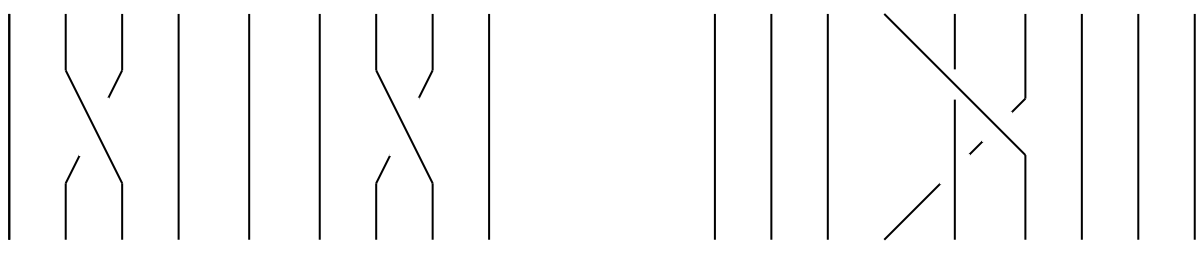

Figure 2. Monomial braids $\rho_{2}$ (left) and $\rho_{0}$ (right) for $r=2$
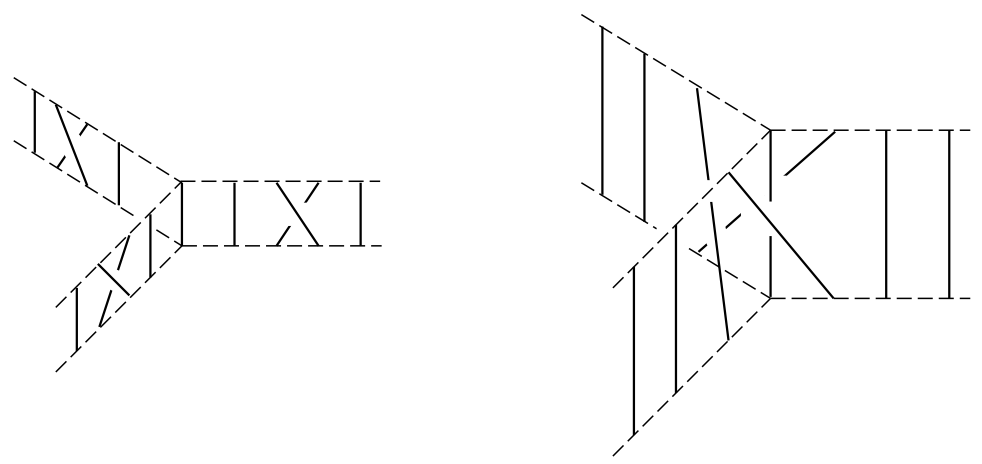

Figure 3. Monomial braids $\rho_{2}$ (left) and $\rho_{0}$ (right) for $r=3$

Expressing these monomial braids in terms of the standard generators $\sigma_{i}$ of the Artin braid group $B_{r n+1}$ is in general fairly difficult. We describe one way to accomplish this.

For each $i$, the strands of the braid $\rho_{i}$ emanate from the points $0, k \zeta^{p} \in \mathbb{C}, 1 \leq k \leq n$, $1 \leq p \leq r$. Order these $r n+1$ strands as follows:

$$
\begin{array}{rcccccccccc}
\text { strand \# : } & 1 & 2 & 3 & \ldots & r & r+1 & r+2 & \ldots & 2 r+1 & \ldots \\
\text { point : } & 0 & \zeta & \zeta^{2} & \ldots & \zeta^{r-1} & \zeta^{r} & 2 \zeta & \ldots & 2 \zeta^{r} & \ldots
\end{array}
$$

For $p=1, \ldots, r-1$, successively rotate the rays $\zeta^{r-p} \cdot \mathbb{R}^{+}$until the initial points of the strands lies on the positive real axis in order. With these choices, the monomial braids are given by

$$
\begin{aligned}
\rho_{0} & =\sigma_{r} \sigma_{r-1} \cdots \sigma_{1} \sigma_{1} \\
\rho_{1} & =\tau_{1}^{-1} \cdot \sigma_{2} \sigma_{4} \cdots \sigma_{2 r} \cdot \tau_{1} \\
\rho_{i} & =\tau_{i}^{-1} \cdot \sigma_{(i-1) r+2} \sigma_{(i-1) r+4} \cdots \sigma_{i r+r} \cdot \tau_{i}, \quad \text { for } 2 \leq i \leq n-1,
\end{aligned}
$$

where

$\tau_{1}=\left(\sigma_{3} \sigma_{5} \cdots \sigma_{2 r-1}\right)\left(\sigma_{4} \sigma_{6} \cdots \sigma_{2 r-2}\right) \cdots\left(\sigma_{r-1} \sigma_{r+1} \sigma_{r+3}\right)\left(\sigma_{r} \sigma_{r+2}\right)\left(\sigma_{r+1}\right)$, and for $i>1$, $\tau_{i}=\left(\sigma_{(i-1) r+3} \sigma_{(i-1) r+5} \cdots \sigma_{i r+r-1}\right) \cdots\left(\sigma_{i r-1} \sigma_{i r+1} \sigma_{i r+3}\right)\left(\sigma_{i r} \sigma_{i r+2}\right)\left(\sigma_{i r+1}\right)$.

Using this, or experimenting with geometric braids, one can show that the monomial braids $\rho_{0}, \rho_{1}, \ldots, \rho_{n-1}$ satisfy the relations

$$
\begin{gathered}
\left(\rho_{0} \rho_{1}\right)^{2}=\left(\rho_{1} \rho_{0}\right)^{2}, \quad \rho_{i} \rho_{i+1} \rho_{i}=\rho_{i+1} \rho_{i} \rho_{i+1} \quad(1 \leq i<n), \quad \text { and } \\
\rho_{i} \rho_{j}=\rho_{j} \rho_{i} \quad(|j-i|>1) .
\end{gathered}
$$

Let $B(r, n)$ denote the group with generators $\rho_{0}, \rho_{1}, \ldots, \rho_{n-1}$ and relations (2.5). This is the (full) monomial braid group, the fundamental group of the quotient space $F_{\Gamma}\left(\mathbb{C}^{*}, n\right) / W$, where $W=G(r, n)$ is the full monomial group, cf. [BMR. Note that $B(r, n)$ is independent of $r$. This group admits natural surjection to $G(r, n)$, which 
may be presented with generators $\rho_{0}, \rho_{1}, \ldots, \rho_{n-1}$ and relations (2.5) together with

$$
\rho_{0}^{r}=\rho_{1}^{2}=\cdots=\rho_{n-1}^{2}=1 .
$$

The pure monomial braid group may be realized as $P(r, n)=\operatorname{ker}(B(r, n) \rightarrow G(r, n))$, the kernel of the aforementioned surjection, see BMR. Elements of $P(r, n)$ are represented by (equivalence classes of) monomial braids $\beta$ as above with the property that $b_{j}(0)=b_{j}(1)$ for all $j$, thus are elements of the fundamental group of the space $S(r, n)$, homeomorphic to the orbit configuration space $F_{\Gamma}\left(\mathbb{C}^{*}, n\right)$. We specify a number of these pure monomial braids.

For $1 \leq i \leq n$, let $X_{i}=\rho_{i-1} \cdots \rho_{2} \rho_{1} \rho_{0} \rho_{1} \rho_{2} \cdots \rho_{i-1}$, and define

$$
\begin{aligned}
Z_{j} & =\rho_{j-1} \cdots \rho_{2} \rho_{1} \rho_{0}^{r} \rho_{1}^{-1} \rho_{2}^{-1} \cdots \rho_{j-1}^{-1}(1 \leq j \leq n), \\
A_{i, j}^{(p)} & =X_{i}^{p-r} \cdot \rho_{j-1} \cdots \rho_{i+1} \rho_{i}^{2} \rho_{i+1}^{-1} \cdots \rho_{j-1}^{-1} \cdot X_{i}^{r-p}(1 \leq i<j \leq n, 1 \leq p \leq r) .
\end{aligned}
$$

Proposition 2.2.1. The pure monomial braids $Z_{j}$ and $A_{i, j}^{(p)}(1 \leq i<j, 1 \leq p \leq r)$ generate the factor $\mathbb{F}_{r(j-1)+1}$ in the realization $P(r, n)=\rtimes_{j=1}^{n} \mathbb{F}_{r(j-1)+1}$ of the pure monomial braid group as an iterated semidirect product of free groups. In particular, these braids (for $1 \leq j \leq n$ ) generate the group $P(r, n)$.

Proof. It suffices to show that the braids $Z_{n}, A_{i, n}^{(p)}$ generate the free group $\mathbb{F}_{r(n-1)+1}=$ $\operatorname{ker}(P(r, n) \rightarrow P(r, n-1))$. Identify the pure monomial braid group with the fundamental group of the space $S(r, n)$.

The free group $\mathbb{F}_{r(n-1)+1}$ is the fundamental group of the fiber of the projection $p_{\Gamma}: F_{\Gamma}\left(\mathbb{C}^{*}, n\right) \rightarrow F_{\Gamma}\left(\mathbb{C}^{*}, n-1\right)$. Via the imbeddings $g_{k}: F_{\Gamma}\left(\mathbb{C}^{*}, k\right) \rightarrow F(\mathbb{C}, r k+1)$, this projection corresponds to the map $S(r, n) \rightarrow S(r, n-1)$ defined by forgetting the last $r$ coordinates. In terms of geometric braids, this map is given by forgetting the last, or outermost, $r$ strands. From this and the definitions of the braids $Z_{n}$ and $A_{i, n}^{(p)}$, it is clear that these braids are elements of $\mathbb{F}_{r(n-1)+1}=\operatorname{ker}(P(r, n) \rightarrow P(r, n-1))$.

Recall from (2.3) that the strands of monomial braids are indexed by the points $0, k \zeta^{p} \in \mathbb{C}$ from which these braids emanate. Checking that the braid $Z_{n}$ performs a full twist on the $r$ strands emanating from $n \zeta, n \zeta^{2}, \ldots, n \zeta^{r}$, and that $A_{i, n}^{(p)}$ simultaneously performs full twists on the pairs of strands emanating from $i \zeta^{p+q}$ and $n \zeta^{q}, 1 \leq q \leq r$, we see that the homology classes of these braids are independent. It follows that the braids $Z_{n}$ and $A_{i, n}^{(p)}$ generate the free group $\mathbb{F}_{r(n-1)+1}$.

In principle, Theorem 2.1.3 and the above result yield a presentation of the group $P(r, n)$ as an iterated semidirect product of free groups, see Remarks 1.1.7 and 1.1.9. See Theorem 1.4.3 for the special case $r=2$. In general, the requisite Artin representation calculations are too complicated to pursue fruitfully. However, using Proposition 1.3.3, we can record some useful qualitative information concerning this presentation. Recall that $\widetilde{U}$ denotes some conjugate of an element $U$ of a free group.

Proposition 2.2.2. In the pure monomial braid group $P(r, n)=\rtimes_{j=1}^{n} \mathbb{F}_{r(j-1)+1}$, for $j<l$, the action of $\mathbb{F}_{r(j-1)+1}$ on $\mathbb{F}_{r(l-1)+1}$ is of the form

$$
\begin{aligned}
Z_{j}^{-1} Z_{l} Z_{j} & =U \widetilde{Z}_{l} U^{-1}, & \left(A_{i, j}^{(p)}\right)^{-1} Z_{l} A_{i, j}^{(p)} & =\widetilde{Z}_{l}, \\
Z_{j}^{-1} A_{k, l}^{(q)} Z_{j} & =V_{k} \widetilde{A}_{k, l}^{(q)} V_{k}^{-1}, & \left(A_{i, j}^{(p)}\right)^{-1} A_{k, l}^{(q)} A_{i, j}^{(p)} & =W_{k} \widetilde{A}_{k, l}^{(q)} W_{k}^{-1},
\end{aligned}
$$

where $U=V_{j}=\widetilde{A}_{j, l}^{(1)} \widetilde{A}_{j, l}^{(2)} \cdots \widetilde{A}_{j, l}^{(r-1)} \widetilde{Z}_{l} \widetilde{A}_{j, l}^{(r)}, V_{k}=1$ if $k \neq j, W_{i}=\widetilde{A}_{i, l}^{(q)} \widetilde{A}_{j, l}^{(m)}$ if $q \equiv p+m$ $\bmod r, W_{j}=\widetilde{A}_{i, l}^{(m)} \widetilde{A}_{j, l}^{(q)}$ if $m \equiv p+q \bmod r$, and $W_{k}=1$ otherwise.

While the conjugates $\widetilde{Z}_{l}$ and $\widetilde{A}_{i, l}^{(q)}$ in the free group $\mathbb{F}_{r(l-1)+1}$ which appear above are not readily accessible, one can use this result and some general facts from the 
theory of hyperplane arrangements to obtain an explicit presentation of the group $P(r, n)$. We briefly recall these facts concerning the "Randell-Arvola" presentation of an arrangement group. See $[\mathrm{Ra}, \mathrm{Ar}, \mathrm{OT}, \mathrm{CS1} \mid$ for detailed discussions.

For a general arrangement $\mathcal{A}=\left\{H_{1}, \ldots, H_{n}\right\}$, the fundamental group $G=G(\mathcal{A})$ of the complement is generated by meridonal loops, $g_{j}$ about $H_{j}$. Relations in the group $G$ arise from codimension two intersections of hyperplanes. If $H_{1} \cap \cdots \cap H_{m}$ is such an intersection, then in $G$ one has a corresponding family of $m-1$ relations

$$
\widetilde{g}_{1} \widetilde{g}_{2} \cdots \widetilde{g}_{m}=\widetilde{g}_{2} \cdots \widetilde{g}_{m} \cdot \widetilde{g}_{1}=\cdots \cdots=\widetilde{g}_{m} \cdot \widetilde{g}_{1} \cdots \widetilde{g}_{m-1} .
$$

This family of (commutation) relations is denoted by $\left[\widetilde{g}_{1}, \ldots, \widetilde{g}_{m}\right]$.

For the monomial arrangement $A_{r, n}$, the codimension two intersections of hyperplanes are recorded implicitly in Proposition 2.2.2. They are, for $j<l$,

$$
\begin{aligned}
& H_{j} \cap H_{j, l}^{(1)} \cap \cdots \cap H_{j, l}^{(r-1)} \cap H_{l} \cap H_{j, l}^{(r)}, \quad H_{j} \cap H_{k, l}^{(q)} \text { if } j \neq k, \quad H_{i, j}^{(p)} \cap H_{l}, \\
& H_{i, j}^{(p)} \cap H_{k, l}^{(q)} \text { if }\{i, j\} \cap\{k, l\}=\emptyset, \quad H_{i, j}^{(p)} \cap H_{i, l}^{(m)} \cap H_{j, l}^{(q)} \text { if } m \equiv p+q \bmod r .
\end{aligned}
$$

While we do not record the conjugations arising in the iterated semidirect product structure of the group $P(r, n)$, we can, with some effort, record those arising in a Randell-Arvola presentation of this group. Recall the monomial braids $X_{i}=$ $\rho_{i-1} \cdots \rho_{2} \rho_{1} \rho_{0} \rho_{1} \rho_{2} \cdots \rho_{i-1}$. For $p<r$, write $A_{i, j}^{[p]}=A_{i, j}^{(p)} A_{i, j}^{(p+1)} \cdots A_{i, j}^{(r-1)}$. Set $A_{i, j}^{[r]}=1$. We require the following technical result. We omit the proof, which is a delicate exercise using the relations (2.5) satisfied by monomial braids and the definition (2.6).

Lemma 2.2.3. We have

$$
X_{i}^{-1} Z_{l} X_{i}= \begin{cases}Z_{l} & \text { if } l<i, \\ C_{l}^{-1} Z_{l} C_{l} & \text { if } l=i, \\ A_{i, l}^{(r-1)} Z_{l}\left(A_{i, l}^{(r-1)}\right)^{-1} & \text { if } l>i,\end{cases}
$$

where $C_{l}=A_{1, l}^{(r)} A_{2, l}^{(r)} \cdots A_{l-1, l}^{(r)}$, and

$$
X_{i}^{-1} A_{k, l}^{(r)} X_{i}= \begin{cases}A_{k, l}^{(r)} & \text { if } i<k \text { or } i>l, \\ A_{k, l}^{(r-1)} & \text { if } i=k, \\ A_{i, l}^{(r-1)} A_{k, l}^{(r)}\left(A_{i, l}^{(r-1)}\right)^{-1} & \text { if } k<i<l, \\ D_{k} A_{k, l}^{(1)} D_{k}^{-1} & \text { if } i=l,\end{cases}
$$

where $D_{k}=A_{k-1, k}^{[1]} A_{k-2, k}^{[1]} \cdots A_{1, k}^{[1]} Z_{k} C_{k}$.

Define elements $Q_{j, p} \in P(r, n)$ by $Q_{1, p}=1$, and for $j \geq 2$,

$$
Q_{j, p}=C_{j} \prod_{q=1}^{p}\left(D_{1} A_{1, j}^{(q)} D_{1}^{-1} D_{2} A_{2, j}^{(q)} D_{2}^{-1} \cdots D_{j-1} A_{j-1, j}^{(q)} D_{j-1}^{-1}\right) .
$$

Theorem 2.2.4. The pure monomial braid group $P(r, n)$ admits a presentation with generators $Z_{j}(1 \leq j \leq n), A_{i, j}^{(p)}(1 \leq i<j \leq n, 1 \leq p \leq r)$, and for $i<j<k<l$, relations

$$
\begin{aligned}
{\left[Z_{j}, Q_{j, r-1} A_{j, l}^{(1)} Q_{j, r-1}^{-1}, \ldots, Q_{j, 1} A_{j, l}^{(r-1)} Q_{j, 1}^{-1}, Z_{l}, A_{j, l}^{(r)}\right], } & \\
{\left[Z_{i}, A_{k, l}^{(p)}\right], \quad\left[A_{i, j}^{(p)}, Z_{k}\right], \quad\left[A_{i, j}^{[p]} Z_{j}\left(A_{i, j}^{[p]}\right)^{-1},\left(A_{i, k}^{(p)}\right)^{\left(A_{i+1, k}^{(r)} \cdots A_{j, k}^{(r)}\right)}\right], } & 1 \leq p \leq r \\
{\left[A_{i, j}^{(p)}, A_{k, l}^{(q)}\right], \quad\left[A_{j, k}^{(p)}, A_{j, l}^{[p]} A_{i, l}^{(q)}\left(A_{j, l}^{[p]}\right)^{-1}\right], } & 1 \leq p, q \leq r \\
{\left[A_{j, k}^{[p]} A_{i, k}^{(q)}\left(A_{j, k}^{[p]}\right)^{-1},\left(A_{j, l}^{(p)}\right)^{(r),}\right], \quad\left[A_{i, j}^{(p)}, A_{i, k}^{(p)}, A_{j, k}^{(r)}\right], } & 1 \leq p, q \leq r \\
{\left[A_{i, j}^{(p)}, A_{j, k}^{(r-q)}, A_{j, k}^{[r-q+1]} A_{i, k}^{(p-q)}\left(A_{j, k}^{[r-q+1]}\right)^{-1}\right], } & 1 \leq q<p \leq r, \\
{\left[D_{i} A_{i, j}^{(p)} D_{i}^{-1}, A_{j, k}^{(r-q)}, A_{j, k}^{[r-q+1]} A_{i, k}^{(r-q+p)}\left(A_{j, k}^{[r-q+1]}\right)^{-1}\right], } & 1 \leq p \leq q<r,
\end{aligned}
$$


Sketch of Proof. It follows from the above discussion that the group $P(r, n)$ admits a presentation of this form. We sketch how one may use Lemma 2.2.3 and the monomial braid relations $(2.5)$ to show that the conjugating words appearing in the relation families (2.7)-2.12) are as asserted.

Using (2.5), one can show that $Z_{1} A_{1,2}^{(1)} \cdots A_{1,2}^{(r-1)} Z_{2} A_{1,2}^{(r)}=\left(\rho_{0} \rho_{1}\right)^{2 r}$ commutes with $Z_{1}, Z_{2}$, and $A_{1,2}^{(p)}$ for each $p$. Relations $(2.7)$ with $(j, l)=(1,2)$ follow. Let $\nu_{2}=\rho_{1} \rho_{2}$ and

$\nu_{j}=\rho_{1} \cdots \rho_{j} \cdot \nu_{j-1}$ for $j>2$. Then $\nu_{j} Z_{1} \nu_{j}^{-1}=Z_{j}$ and $\nu_{j} Z_{2} \nu_{j}^{-1}=Z_{j+1}$. Conjugating (2.7) with $(j, l)=(1,2)$ by $\nu_{j}$ yields (2.7) for any $j$ and $l=j+1$. Conjugating these last relations by $\rho_{l} \cdots \rho_{j+1}$ yields (2.7) in general.

Similarly, using (2.5), one can show that the following relations hold in $P(r, n)$ :

$$
\left[Z_{i}, A_{j, k}^{(r)}\right], \quad\left[Z_{j},\left(A_{i, k}^{(r)}\right)^{\left(A_{i+1, k}^{(r)} \cdots A_{j, k}^{(r)}\right)}\right], \quad \text { and } \quad\left[A_{i, j}^{(r)}, Z_{k}\right] .
$$

Repeated conjugation these relations by $X_{t}, t=i, j, k$, and use of Lemma 2.2.3 yields the relations (2.8).

Finally, it is clear from the definition (2.6) that the pure monomial braids $A_{i, j}^{(r)}$ satisfy the classical pure braid relations (1.4). These relations may be rewritten as

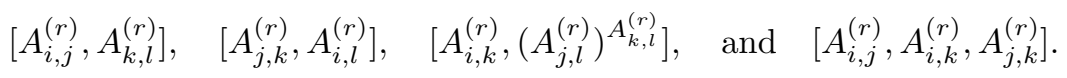

Repeated conjugation these relations by $X_{t}, t=i, j, k, l$, and use of Lemma 2.2.3 yields the relations 2.9)-(2.12).

Remark 2.2.5. In the special case $r=2$, one can check that the presentations of the generalized pure braid group $P B_{n}=P(2, n)$ given in Theorems 1.4.3 and 2.2.4 are equivalent. The correspondence between the generators in these two results is given by $a_{i, j}=A_{i, j}^{(2)}, b_{i, j}=A_{i, j}^{(1)}$, and $c_{j}=Z_{j}$.

\subsection{The Lie Algebra Associated to the Lower Central Series.}

We now show how the results of the previous section may be used to determine the structure of the Lie algebra associated to the lower central series of the pure monomial braid group.

For any group $G$, let $G_{k}$ denote the $k$-th lower central series subgroup, defined inductively by $G_{1}=G$ and $G_{k+1}=\left[G_{k}, G\right]$ for $k \geq 1$. Let $\operatorname{gr} G=\bigoplus_{k>1} G(k)$, where $G(k)=G_{k} / G_{k+1}$. The map (of sets) $G \times G \rightarrow G$ given by the commutator, $(x, y) \mapsto[x, y]=x y x^{-1} y^{-1}$, induces a bilinear map $\operatorname{gr} G \times \operatorname{gr} G \rightarrow \operatorname{gr} G$ which defines a Lie algebra structure on $\operatorname{gr} G$, see for instance $[\mathrm{Se}$, Chapter I].

Example 2.3.1. If $G=\mathbb{F}_{n}$ is a finitely generated free group, then $\operatorname{gr} G$ is isomorphic to the free Lie algebra $L(n)$ on $n$ generators, see [Se, Chapter IV].

The additive structure of the Lie algebra associated to the lower central series of the fundamental group of the complement of a fiber-type arrangement is given by the following result.

Theorem 2.3.2 (Falk and Randell |FR1], Theorem 3.1). Let $1 \rightarrow H \stackrel{i}{\rightarrow} G \stackrel{j}{\rightarrow} K \rightarrow 1$ be a split extension of groups such that $K$ acts trivially on $H_{1} / H_{2}$. Then the induced sequence of graded abelian groups, $0 \rightarrow \operatorname{gr} H \rightarrow \operatorname{gr} G \rightarrow \operatorname{gr} K \rightarrow 0$, is split exact.

In the case where $G$ is the group of a fiber-type arrangement, the proof of this result in FR1] shows that the lower central series quotients $G(k)$ are free abelian for all $k$, see [FR2]. Applying this result and observation inductively, we obtain

Corollary 2.3.3. Let $\mathcal{A}$ be a fiber-type arrangement with exponents $\left\{d_{1}, \ldots, d_{\ell}\right\}$ and group $G$. Then, as abelian groups, gr $G \cong L\left(d_{1}\right) \oplus L\left(d_{2}\right) \oplus \cdots \oplus L\left(d_{\ell}\right)$. 
We now pursue the Lie bracket relations in $\operatorname{gr} G$ for these groups, in particular for the pure monomial braid groups $P(r, n)$.

In the situation of Theorem 2.3.2, the group $G \cong H \rtimes_{\alpha} K$ may be realized as the semidirect product of $H$ and $K$, determined by a homomorphism $\alpha: K \rightarrow \operatorname{Out}(H)$. If $s: K \rightarrow G$ denotes the splitting, then $G$ is generated by $i(H)$ and $s(K)$. Identifying $H$ and $K$ with their images in $G$, we have relations $x^{-1} y x=\alpha(x)(y)$ in $G$ for $y \in H$ and $x \in K$. If the action of $K$ on $H$ is by conjugation, the Lie bracket relations in gr $G$ are readily obtained from the relations in $G$ itself, as follows.

Lemma 2.3.4. Let $y \in H$ and $x \in K$. If $x^{-1} y x=w y w^{-1}$ for some $w \in H$, then $[\bar{x}+\bar{w}, \bar{y}]=0$ in $\operatorname{gr} G$, where $\bar{x}, \bar{y}$, and $\bar{w}$ are the images of $x, y$, and $w$ in $\operatorname{gr} G$.

Proof. Rewriting $x^{-1} y x=w y w^{-1}$ as $1_{G}=x w y w^{-1} x^{-1} y^{-1}=[x w, y] \in G_{2}$, the result follows from the commutator identity $[x w, y]=[x,[w, y]][w, y][x, y]$ in $G$.

We subsequently write simply $g$, as opposed to $\bar{g}$, for the image in gr $G$ of $g \in G$.

Example 2.3.5. The structure of the Lie algebra associated to the lower central series of the classical pure braid group $P_{n}=\rtimes_{j=1}^{n-1} \mathbb{F}_{j}$ was determined by Kohno $[\mathrm{K} 2$. The above considerations may be used to recover this result. By Corollary 2.3.3, we have gr $P_{n} \cong \bigoplus_{j=1}^{n-1} L(j)$, where the free Lie algebra $L(j)$ is generated by $A_{1, j+1}, \ldots, A_{j, j+1}$. Applying Lemma 2.3.4 to the defining relations (1.4) of $P_{n}$, we see that the Lie bracket relations in gr $P_{n}$ are the "infinitesimal pure braid relations," given by

$$
\begin{aligned}
{\left[A_{i, j}+A_{i, k}+A_{j, k}, A_{m, k}\right] } & =0 & & \text { for } m=i, j, \\
{\left[A_{i, j}, A_{k, l}\right] } & =0 & & \text { for }\{i, j\} \cap\{k, l\}=\emptyset .
\end{aligned}
$$

The Lie algebra $\operatorname{gr} P_{n}$ arises in a number of other contexts. For instance, the integral homology, $H_{*}\left(\Omega F\left(\mathbb{C}^{k}, n\right)\right)$, of the loop space of the classical configuration space was recently computed by Fadell and Husseini $[\mathrm{FH}]$. Subsequently, Cohen and Gitler [CG] showed that, with appropriate regrading, the Lie algebra of primitives, $P H_{*}\left(\Omega F\left(\mathbb{C}^{k}, n\right)\right)$, is isomorphic to gr $P_{n}$ for $k \geq 2$.

The orbit configuration spaces $F_{\Gamma}\left(\mathbb{C}^{k} \backslash\{0\}, n\right)$, where $\Gamma=\mathbb{Z} / r \mathbb{Z}$ acts on $\mathbb{C}^{k} \backslash\{0\}$ by multiplication by a primitive $r$-th root of unity, provide natural generalizations of these results. In the case $k=1$, using Theorem 2.2.4 (or Proposition 2.2.2), we obtain:

Theorem 2.3.6. Let $\operatorname{gr} P(r, n)$ be the Lie algebra associated to the lower central series of the pure monomial braid group. Then, gr $P(r, n) \cong \bigoplus_{j=0}^{n-1} L(r j+1)$ as abelian groups, where $L(r j+1)$ is generated by $Z_{j+1}$ and $A_{i, j+1}^{(p)}, 1 \leq i \leq j, 1 \leq p \leq r$. The Lie bracket relations in gr $P(r, n)$ are given by

$$
\begin{array}{rlrl}
{\left[Z_{j}+Z_{l}+A_{j, l}^{(1)}+A_{j, l}^{(2)}+\cdots+A_{j, l}^{(r)}, Y\right]} & =0 & & \text { for } Y=Z_{l}, Y=A_{j, l}^{(p)}, 1 \leq p \leq r, \\
{\left[A_{i, j}^{(p)}+A_{i, k}^{(q)}+A_{j, k}^{(m)}, Y\right]=0} & & \text { for } Y=A_{i, k}^{(q)}, A_{j, k}^{(m)}, q \equiv p+m \bmod r, \\
{\left[A_{i, j}^{(p)}, A_{k, l}^{(q)}\right]=0} & & \text { for }\{i, j\} \cap\{k, l\}=\emptyset, 1 \leq p, q \leq r, \text { and } \\
{\left[Z_{k}, A_{i, j}^{(p)}\right]=0} & & \text { for } k \neq i, j \text { and } 1 \leq p \leq r .
\end{array}
$$

For $k \geq 2$, consider the loop space $\Omega F_{\Gamma}\left(\mathbb{C}^{k} \backslash\{0\}, n\right)$. The Lie algebra of primitives in the homology, $H_{*}\left(\Omega F_{\Gamma}\left(\mathbb{C}^{k} \backslash\{0\}, n\right)\right)$, was calculated by Xicoténcatl [Xi, Theorem 3.1.2]. Comparing the Lie bracket relations obtained there and those in $\operatorname{gr} P(r, n)$ recorded above, we obtain the following, which was conjectured in [Xi, Section 3.7].

Corollary 2.3.7. For $k \geq 2$, with appropriate regrading, the Lie algebra $\operatorname{gr} P(r, n)$ is isomorphic to the Lie algebra of primitives, $P H_{*}\left(\Omega F_{\Gamma}\left(\mathbb{C}^{k} \backslash\{0\}, n\right)\right)$, in the Hopf algebra $H_{*}\left(\Omega F_{\Gamma}\left(\mathbb{C}^{k} \backslash\{0\}, n\right)\right)$. 
Similar considerations reveal the structure of the Lie algebra associated to the lower central series of the fundamental group of the complement of an arbitrary fiber-type arrangement $\mathcal{A}$ defined by $Q(\mathcal{A})=\prod_{j=1}^{\ell} Q_{j}\left(x_{1}, \ldots, x_{j}\right)$. Recall the presentation of the group $G$ of such an arrangement from Remark 1.1.9, with generators $x_{q, j}$ and relations $x_{p, i}^{-1} x_{q, j} x_{p, i}=\eta_{j}\left(x_{p, i}\right)\left(x_{q, j}\right)$. The generators $x_{q, j}$ correspond to the hyperplanes $H_{q, j}$ defined by the degree $d_{j}$ polynomial $Q_{j}\left(x_{1}, \ldots, x_{j}\right)$, and $\eta_{j}=\alpha_{d_{j}} \circ \gamma_{j}$ is the composition of the Artin representation and the homomorphism induced by the map $g_{j}$ of (1.6).

From the identification of the monodromy of the strictly linearly fibered bundle $M\left(\mathcal{A}_{j}\right) \rightarrow M\left(\mathcal{A}_{j-1}\right)$ in Section 1.3, for $i<j$ we have $\gamma_{j}\left(x_{p, i}\right)=\prod_{k=1}^{r} A_{V_{k}}^{\zeta_{k}}$, where $V=\left(V_{1}\left|V_{2}\right| \cdots \mid V_{r}\right)$ is the partition of $\left[d_{j}\right]$ recording the hyperplanes of $\mathcal{A}_{j} \backslash \mathcal{A}_{j-1}$ which meet $H_{p, i}$ in codimension two, cf. Theorem 1.3.1 and Remark 1.3.2. Applying the Artin representation as in Proposition 1.3.3, the relations in $G$ may be expressed more explicitly as

$$
x_{p, i}^{-1} \cdot t_{q} \cdot x_{p, i}=\widetilde{t}_{V_{k}} \cdot \widetilde{t}_{q} \cdot \tilde{t}_{V_{k}}^{-1}
$$

where $t_{m}=x_{m, j}$ for $1 \leq m \leq d_{j}$ and $V_{k}$ is the unique block of $V$ containing $q$.

Theorem 2.3.8. Let $\mathcal{A}$ be a fiber-type arrangement with exponents $\left\{d_{1}, \ldots, d_{\ell}\right\}$, and group $G=G(\mathcal{A})$. Then, gr $G \cong \bigoplus_{j=1}^{\ell} L\left(d_{j}\right)$ as abelian groups, where $L\left(d_{j}\right)$ is generated by $x_{q, j}, 1 \leq p \leq d_{j}, 1 \leq j \leq \ell$. The Lie bracket relations in $\operatorname{gr} G$ are given by

$$
\left[x_{p, i}+x_{q_{1}, j}+x_{q_{2}, j}+\cdots+x_{q_{m}, j}, x_{q_{k}, j}\right]=0 \quad \text { for } 1 \leq k \leq m,
$$

where $1 \leq i<j \leq \ell$ and $\left\{q_{1}, \ldots, q_{m}\right\}$ is maximal such that codim $H_{p, i} \cap \bigcap_{k=1}^{m} H_{q_{k}, j}=2$.

Proof. The isomorphism, gr $G \cong \bigoplus_{j=1}^{\ell} L\left(d_{j}\right)$, of graded abelian groups was noted in Corollary 2.3.3 above. The Lie bracket relations in $\operatorname{gr} G$ may be obtained by applying Lemma 2.3.4 to the relations (2.13).

For any arrangement $\mathcal{A}$ of $n$ hyperplanes, the (rational) holonomy Lie algebra $\mathcal{L}_{\mathbb{Q}}$ of the complement $M$ is the quotient of the free Lie algebra $L_{\mathbb{Q}}(n)=L\left(H_{1}(M ; \mathbb{Q})\right)$ by the image of the map $H_{1}(M ; \mathbb{Q}) \rightarrow \bigwedge^{2} H_{1}(M ; \mathbb{Q})$ dual to the cup product. For a fiber-type arrangement with exponents $\left\{d_{1}, \ldots, d_{\ell}\right\}$, Jambu $\left[\mathrm{Ja}\right.$ shows that $\mathcal{L}_{\mathbb{Q}} \cong \bigoplus_{j=1}^{\ell} L_{\mathbb{Q}}\left(d_{j}\right)$ as graded vector spaces. For an arbitrary arrangement, Kohno [K1] shows that $\mathcal{L}_{\mathbb{Q}}$ is generated by elements $x_{1}, \ldots, x_{n}$ in one-to-one correspondence with the hyperplanes of $\mathcal{A}$, with relations

$$
\left[x_{q_{1}}+x_{q_{2}}+\cdots+x_{q_{m}}, x_{q_{k}}\right]=0, \quad 1 \leq k \leq m,
$$

for each maximal family $\left\{H_{q_{1}}, \ldots, H_{q_{m}}\right\}$ of hyperplanes of $\mathcal{A}$ with codim $\bigcap_{k=1}^{m} H_{q_{k}}=2$.

In light of this result, by imposing the relations (2.15) on the free Lie algebra $L(n)=L_{\mathbb{Z}}(n)=L\left(H_{1}(M ; \mathbb{Z})\right)$, we may consider the integral holonomy Lie algebra $\mathcal{L}=\mathcal{L}_{\mathbb{Z}}$ of $M$. Furthermore, comparing the relations 2.14) and (2.15) for a fiber-type arrangement, we have the following result, which may also be obtained from the work of Kohno [K1, K2, together with the results of Falk and Randell stated above.

Corollary 2.3.9. Let $\mathcal{A}$ be a fiber-type arrangement with complement $M$ and group $G$. Then the integral holonomy Lie algebra $\mathcal{L}$ of $M$ is isomorphic to the Lie algebra $\operatorname{gr} G$ associated to the lower central series of $G$.

Acknowledgments. Portions of this work were carried during visits to the Department of Mathematics at the University of Wisconsin-Madison in the Spring of 1999. We thank the Department for its hospitality, and the College of Arts \& Sciences at Louisiana State University for granting the Research Fellowship which made these visits possible. We also thank Fred Cohen and Peter Orlik for useful conversations. Some of the results presented here stem from our collaboration with Alex Suciu, who we acknowledge. 


\section{REFERENCES}

[AFR] C.S. Aravinda, F.T. Farrell, S.K. Roushon, Algebraic K-theory of pure braid groups, Asian J. Math. (to appear).

[Ar] W. Arvola, The fundamental group of the complement of an arrangement of complex hyperplanes, Topology 31 (1992), 757-766.

[Bi] J. Birman, Braids, Links and Mapping Class Groups, Ann. of Math. Studies, vol. 82, Princeton Univ. Press, Princeton, NJ, 1975.

[Br] E. Brieskorn, Sur les groupes de tresses (d'après V. I. Arnol'd), In: Séminaire Bourbaki, 24ème année (1971/1972), Exp. No. 401, Lect. Notes in Math., vol. 317, Springer-Verlag, Berlin, 1973, pp. $21-44$.

[BMR] M. Broué, G. Malle, R. Rouquier, Complex reflection groups, braid groups, Hecke algebras, J. reine angew. Math. 500 (1998), 127-190.

[CS1] D. Cohen, A. Suciu, The braid monodromy of plane algebraic curves and hyperplane arrangements, Comment. Math. Helv. 72 (1997), 285-315.

[CS2] Homology of iterated semidirect products of free groups, J. Pure Appl. Algebra 126 (1998), 87-120.

[CG] F. Cohen, S. Gitler, On loop spaces of configuration spaces, preprint 1998.

[FH] E. Fadell, S. Husseini, Geometry and Topology of Configuration Spaces, in preparation.

[FN] E. Fadell, L. Neuwirth, Configuration spaces, Math. Scand. 10 (1962), 119-126.

[FR1] M. Falk, R. Randell, The lower central series of a fiber-type arrangement, Invent. Math. 82 (1985), 77-88.

[FR2] , Pure braid groups and products of free groups, in: Braids, Contemp. Math., vol. 78, Amer. Math. Soc., Providence, RI, 1988, pp. 217-228.

[FT] M. Falk, H. Terao, $\beta$ nbc-bases for cohomology of local systems on hyperplane complements, Trans. Amer. Math. Soc. 349 (1997), 189-202.

[Ha] V.L. Hansen, Braids and Coverings, London Math. Soc. Stud. Texts, vol. 18, Cambridge Univ. Press, Cambridge, 1989.

[Ja] M. Jambu, Fiber-type arrangements and factorization properties, Adv. Math. 80 (1990), 1-21.

[K1] T. Kohno, On the holonomy Lie algebra and the nilpotent completion of the fundamental group of the complement of hypersurfaces, Nagoya Math. J. 92 (1983), 21-37.

[K2] - Series de Poincaré-Koszul associée aux groupes de tresses pures, Invent. Math. 82 (1985), 57-75.

[Le] A. Leibman, Fiber bundles with degenerations and their applications to computing fundamental groups, Geom. Dedicata 48 (1993), 93-126.

[OT] P. Orlik, H. Terao, Arrangements of Hyperplanes, Grundlehren Math. Wiss., Vol. 300, Springer-Verlag, Berlin, 1992

[Pa] L. Paris, On the fundamental group of the complement of a complex hyperplane arrangement, in: Singularities and Arrangements, Sapporo-Tokyo, 1998, Adv. Stud. Pure Math., Math. Soc. Japan, to appear.

[Ra] R. Randell, The fundamental group of the complement of a union of complex hyperplanes, Invent. Math. 69 (1982), 103-108. Correction, Invent. Math. 80 (1985), 467-468.

[Se] J.-P. Serre, Lie Algebras and Lie Groups, 2nd ed., Lect. Notes in Math., vol. 1500, SpringerVerlag, Berlin, 1992.

[Xi] M. Xicoténcatl, Orbit configuration spaces, Thesis, University of Rochester, 1997.

Department of Mathematics, Louisiana State University, Baton Rouge, LA 70803

E-mail address: cohen@math.lsu.edu

URL: http://math.lsu.edu/ ${ }^{\sim}$ cohen 